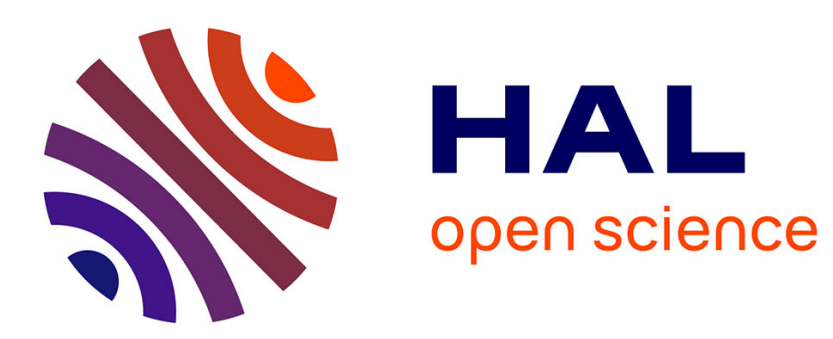

\title{
Robust residual a posteriori error estimators for the Reissner-Mindlin eigenvalues system
}

\author{
Emmanuel Creusé, Serge Nicaise, Emmanuel Verhille
}

\section{To cite this version:}

Emmanuel Creusé, Serge Nicaise, Emmanuel Verhille. Robust residual a posteriori error estimators for the Reissner-Mindlin eigenvalues system. Journal of Numerical Mathematics, 2013, 21 (2), pp.89-134. 10.1515/jnum-2013-0004 . hal-00777682v2

\section{HAL Id: hal-00777682 \\ https://hal.science/hal-00777682v2}

Submitted on 2 Apr 2013

HAL is a multi-disciplinary open access archive for the deposit and dissemination of scientific research documents, whether they are published or not. The documents may come from teaching and research institutions in France or abroad, or from public or private research centers.
L'archive ouverte pluridisciplinaire HAL, est destinée au dépôt et à la diffusion de documents scientifiques de niveau recherche, publiés ou non, émanant des établissements d'enseignement et de recherche français ou étrangers, des laboratoires publics ou privés. 


\title{
Robust residual a posteriori error estimators for the Reissner-Mindlin eigenvalues system
}

\author{
Emmanuel Creusé, Serge Nicaiseł Emmanuel Verhille
}

March 28, 2013

\begin{abstract}
We consider a conforming finite element approximation of the Reissner-Mindlin eigenvalue system, for which a robust a posteriori error estimator for the eigenvector and the eigenvalue errors is proposed. For that purpose, we first perform a robust $a$ priori error analysis without strong regularity assumption. Upper and lower bounds are then obtained up to higher order terms that are superconvergent, provided that the eigenvalue is simple. The convergence rate of the proposed estimator is confirmed by a numerical test.
\end{abstract}

Key Words Reissner-Mindlin plate, finite elements, a posteriori error estimators, eigenvalues.

AMS (MOS) subject classification 74K20; 65M60; 65M15; 65M50.

\section{Introduction}

Nowadays, a posteriori error estimators have become an indispensable tool in the context of finite element methods. They are now widely used in order to control the numerical error, as well as to drive the adaptive mesh refinement processes. Many works have been devoted to this topic (see e.g. [1, 4, 33, 36] for general monographies). Considering the Reissner-Mindlin system, several kind of suitable finite elements exist, and a well known task to overcome is to avoid the so-called "shear locking effect", by using properly defined operators at the discrete level. In the literature, if a lot of papers have already been devoted to the a priori error analysis of this system, far less references can be found on its a

* Laboratoire Paul Painlevé UMR 8524 and INRIA Lille Nord Europe, Université de Lille 1, Cité Scientifique, 59655 Villeneuve d'Ascq Cedex, emmanuel.creuse@math.univ-lille1.fr

${ }^{\dagger}$ LAMAV, FR CNRS 2956, Université de Valenciennes et du Hainaut Cambrésis, , Institut des Sciences et Techniques de Valenciennes, F-59313 - Valenciennes Cedex 9 France, serge.nicaise@univ-valenciennes.fr

¥Université des Sciences et Technologies de Lille, Laboratoire Paul Painlevé, UMR CNRS 8524, Cité Scientifique, 59655 Villeneuve d'Ascq Cedex, emmanuel.verhille@math.univ-lille1.fr (corresponding author) 
posteriori error analysis (see e.g. $[6,9,10,11,20,26,30,32]$ ).

In this work, we are specifically interested in the Reissner-Mindlin eigenvalues system, corresponding to the modeling of a vibration plate problem. Our goal is to derive an a posteriori estimator which is robust with respect to the plate thickness parameter $t$, efficient and also explicitly computable. To our best knowledge, only the a priori analysis of this eigenvalue problem in a regular context is up to now available (see [16, 17, 18, 22, 25, 31] for an overview on this topic). We have here in mind to extend it to the non regular context, and, with these results in hand, to provide a relevant a posteriori error estimator. For similar results for the Laplace equation, we refer to $[29,19]$.

The outline of the paper is as follows : In Section 1, we recall the Reissner-Mindlin eigenvalues system and its discretization. Section 2 gives an a priori error analysis whithout strong regularity assumptions, that constitutes its originality. Section 3 is devoted to some preliminary results in order to prove the upper bound of the a posteriori estimator. This one directly follows and is detailed in section 4 . We then give an a posteriori estimate for the eigenvalues error in section 5. The lower bound is developped in section 6 and leads to the efficiency of our estimator. Finally, some numerical tests are presented in section 7 , that confirm its requested behavior.

\section{The boundary value problem and its discretization}

Let $\tilde{\Omega}$ be a bounded open domain of $\mathbb{R}^{2}$ with a Lipschitz boundary that we suppose to be polygonal. Assuming that the plate is clamped, its free vibration modes are solutions of the following problem (called Reissner-Mindlin eigenvalue problem) : Given $\tilde{t}$ a fixed positive real number that represents the thickness of the plate, find a non-trivial solution $(\tilde{\omega}, \tilde{\phi}) \in H_{0}^{1}(\tilde{\Omega}) \times H_{0}^{1}(\tilde{\Omega})^{2}$ and $\tilde{\nu}_{\tilde{t}}>0$ such that for all $(\tilde{v}, \tilde{\psi}) \in H_{0}^{1}(\tilde{\Omega}) \times H_{0}^{1}(\tilde{\Omega})^{2}$ we have :

$$
\tilde{t}^{3} \tilde{a}(\tilde{\phi}, \tilde{\psi})+\tilde{\zeta} \tilde{t} \int_{\tilde{\Omega}}(\tilde{\nabla} \tilde{\omega}-\tilde{\phi}) \cdot(\tilde{\nabla} \tilde{v}-\tilde{\psi}) d \tilde{x}=\tilde{\nu}_{\tilde{t}}^{2}\left[\tilde{t} \int_{\tilde{\Omega}} \tilde{\rho} \tilde{\omega} \tilde{v} d \tilde{x}+\frac{\tilde{t}^{3}}{12} \int_{\tilde{\Omega}} \tilde{\rho} \tilde{\phi} \cdot \tilde{\psi} d \tilde{x}\right],
$$

where $\tilde{\nu}_{\tilde{t}}$ is the angular vibration frequency, $\tilde{\rho}$ is the density of the plate and

$$
\tilde{a}(\tilde{\phi}, \tilde{\psi})=\int_{\tilde{\Omega}} \tilde{\mathcal{C}} \tilde{\varepsilon}(\tilde{\phi}): \tilde{\varepsilon}(\tilde{\psi}) d \tilde{x}
$$

Here, the operator : denotes the term-by term tensor product and

$$
\tilde{\varepsilon}(\tilde{\phi})=\frac{1}{2}\left(\tilde{\nabla} \tilde{\phi}+(\tilde{\nabla} \tilde{\phi})^{T}\right)
$$

where $\tilde{\nabla}$ denotes the usual gradient operator over $\tilde{\Omega}$. $\tilde{\mathcal{C}}$ is the elasticity tensor given by

$$
\tilde{\mathcal{C}} \tilde{\varepsilon}(\tilde{\phi})=\frac{E}{12(1+\nu)} \tilde{\varepsilon}(\tilde{\phi})+\frac{E \nu}{12\left(1-\nu^{2}\right)} \operatorname{tr}(\tilde{\varepsilon}(\tilde{\phi})) \mathcal{I},
$$


where $E$ and $\nu$ are respectively the Young modulus and the the Poisson coefficient of the material. We also define

$$
\tilde{\zeta}=\frac{E k}{2(1+\nu)},
$$

where $k$ is the shear correction factor usually equal to $5 / 6$ [17]. Now, in order to perform an a posteriori error analysis that do not depend on the chosen unit of length problem (1) has to be given in its dimensionless formulation. To do it, we introduce a density as well as a length scale of reference, respectively denoted by $\bar{\rho}$ and $L$ (the latest being in the order of the diameter of the domain $\tilde{\Omega}$ ). We consequently define the dimensionless variables and unknowns $x, \rho, \phi$ and $\omega$ by :

$$
\tilde{x}=L x, \quad \tilde{\rho}=\bar{\rho} \rho, \quad \tilde{\phi}=\phi \quad \text { and } \quad \tilde{\omega}=L \omega .
$$

Considering the case of the constant density $(\tilde{\rho} \equiv \bar{\rho}$ so that $\rho \equiv 1)$, problem $(1)$ in which the eigenvector is normalized is now equivalent to find a non-trivial $(\omega, \phi) \in H_{0}^{1}(\Omega)^{2} \times H_{0}^{1}(\Omega)$ and $\alpha_{t}>0$ such that for all $(v, \psi) \in H_{0}^{1}(\Omega) \times H_{0}^{1}(\Omega)^{2}$ we have :

$$
\left\{\begin{array}{l}
a(\phi, \psi)+\int_{\Omega} \gamma \cdot(\nabla v-\psi) d x=\alpha_{t}\left[\int_{\Omega} \omega v d x+\frac{t^{2}}{12} \int_{\Omega} \phi \cdot \psi d x\right], \\
\int_{\Omega} \omega^{2} d x+\frac{t^{2}}{12} \int_{\Omega} \phi \cdot \phi d x=1,
\end{array}\right.
$$

where we note :

$$
\begin{gathered}
a(\phi, \psi)=\int_{\Omega} \mathcal{C} \varepsilon(\phi): \varepsilon(\psi) d x, \\
\mathcal{C} \varepsilon(\phi)=2 \mu \varepsilon(\phi)+\lambda \operatorname{tr}(\varepsilon(\phi)) \mathcal{I},
\end{gathered}
$$

with

$$
\mu=\frac{1}{24(1+\nu)}, \quad \lambda=\frac{\nu}{12\left(1-\nu^{2}\right)}
$$

and

$$
\varepsilon(\phi)=\frac{1}{2}\left(\nabla \phi+(\nabla \phi)^{T}\right)
$$

Defining $\zeta=\tilde{\zeta} / E$, the dimensionless variables and parameters arising in (2) are given by :

$$
t=\tilde{t} / L, \quad \gamma=\frac{\zeta}{t^{2}}(\nabla \omega-\phi) \quad \text { and } \quad \alpha_{t}=\frac{\bar{\rho} \tilde{\nu}_{\tilde{t}}^{2} L^{2}}{E t^{2}}
$$

From now on, the parameter $t$ is supposed to be in the interval $\left(0, t_{\max }\right]$ with $t_{\max }>0$ fixed. In the following, $(\cdot, \cdot)_{D}$ stands for the usual inner product in (any power of) $L^{2}(D)$. For shortness the $L^{2}(D)$-norm is denoted by $\|\cdot\|_{D}$. For $s \geq 0$, the usual norm and seminorm of $H^{s}(D)$ are respectively denoted by $\|\cdot\|_{s, D}$ and $|\cdot|_{s, D}$ and the usual norm on $H^{-s}(D)=\left(H_{0}^{s}(\Omega)\right)^{\prime}$ is denoted $\|\cdot\|_{-s, D}$. For all these notations, in the case $D=\Omega$, the 
index $\Omega$ is dropped. The usual Poincaré-Friedrichs constant in $\Omega$ is the smallest positive constant $c_{F}$ such that

$$
\|\phi\| \leq c_{F}|\phi|_{1} \quad \forall \phi \in H_{0}^{1}(\Omega)^{2} .
$$

By Korn's inequality [21], $a$ is an inner product on $H_{0}^{1}(\Omega)^{2}$ equivalent to the usual one. Indeed, defining the energy norm $\|\cdot\|_{\mathcal{C}}$ by

$$
\|\psi\|_{\mathcal{C}}^{2}=a(\psi, \psi) \forall \psi \in H_{0}^{1}(\Omega)^{2},
$$

it can be shown (see [12]) that

$$
|\psi|_{1}^{2} \leq \frac{1}{\mu}\|\psi\|_{\mathcal{C}}^{2} \forall \psi \in H_{0}^{1}(\Omega)^{2}
$$

Let us now consider a discretization of (2) based on a conforming triangulation $\mathcal{T}_{h}$ of $\Omega$ composed of triangles. We assume that this triangulation is regular, i.e., for any element $T \in \mathcal{T}_{h}$, the ratio $h_{T} / \rho_{T}$ is bounded by a constant $\sigma>0$ independent of $T$ and of the mesh size $h=\max _{T \in \mathcal{T}_{h}} h_{T}$, where $h_{T}$ is the diameter of $T$ and $\rho_{T}$ the diameter of its largest inscribed ball. We consider on this triangulation classical conforming finite element spaces $W_{h} \times \Theta_{h}$ such that

$$
\begin{gathered}
W_{h} \subset W_{\ell, h}:=\left\{v_{h} \in \mathcal{C}^{0}(\bar{\Omega}) ; v_{h}=0 \text { on } \partial \Omega \text { and } v_{h \mid T} \in \mathbb{P}_{\ell}(T) \forall T \in \mathcal{T}_{h}\right\} \subset H_{0}^{1}(\Omega), \\
\Theta_{h} \subset W_{\ell, h} \times W_{\ell, h} \subset H_{0}^{1}(\Omega) \times H_{0}^{1}(\Omega),
\end{gathered}
$$

for some positive integer $\ell$, where $\mathbb{P}_{\ell}(T)$ is the space of polynomials of degree at most $l$ defined on $T$. The discrete formulation of the Reissner-Mindlin eigenvalue problem is now to find $\left(\omega_{h}, \phi_{h}\right) \in W_{h} \times \Theta_{h}$ and $\alpha_{t, h}>0$ such that

$$
\left\{\begin{array}{l}
a\left(\phi_{h}, \psi_{h}\right)+\left(\gamma_{h}, \nabla v_{h}-\mathbf{R}_{h} \psi_{h}\right)=\alpha_{t, h}\left[\left(\omega_{h}, v_{h}\right)+\frac{t^{2}}{12}\left(\phi_{h}, \psi_{h}\right)\right], \forall\left(v_{h}, \psi_{h}\right) \in W_{h} \times \Theta_{h}, \\
\left\|\omega_{h}\right\|^{2}+\frac{t^{2}}{12}\left\|\phi_{h}\right\|^{2}=1,
\end{array}\right.
$$

with

$$
\gamma_{h}=\zeta t^{-2}\left(\nabla \omega_{h}-\mathbf{R}_{h} \phi_{h}\right)
$$

Here, $\mathbf{R}_{h}$ denotes the reduction integration operator in the context of shear-locking with values in the so-called discrete shear force space $\Gamma_{h}$ which depends on the involved finite element $[5,7,16,15,35]$. We assume moreover that

$$
\mathbf{R}_{h} \psi_{h} \in H_{0}(\text { rot }, \Omega) \forall \psi_{h} \in \Theta_{h},
$$

where $H_{0}($ rot,$\Omega)=\left\{v \in L^{2}(\Omega)^{2} ;\right.$ rot $v \in L^{2}(\Omega)$ and $v \cdot \tau=0$ on $\left.\partial \Omega\right\}$, equipped with the norm

$$
\|v\|_{H(r o t, \Omega)}^{2}=\|v\|^{2}+\|\operatorname{rot} v\|^{2} .
$$


Here, for any $v=\left(v_{1}, v_{2}\right)^{T} \in L^{2}(\Omega)^{2}, \quad \operatorname{rot} v=\partial v_{2} / \partial x-\partial v_{1} / \partial y$ and $\tau$ is the unit tangent vector along $\partial \Omega$. In this work, $\mathbf{R}_{h}$ is defined as the interpolation operator from $\Theta_{h}$ on the $H_{0}($ rot,$\Omega)$ conforming lower-order Nedelec finite element space [21].

In this paper, we consider the lowest order MITC element (also called the Duran Liberman element) for which $W_{h}$ and $\Theta_{h}$ are defined by

$$
\begin{gathered}
W_{h}=\left\{v_{h} \in \mathcal{C}^{0}(\bar{\Omega}) ; v_{h}=0 \text { on } \partial \Omega \text { and } v_{h \mid T} \in \mathbb{P}_{1}(T) \forall T \in \mathcal{T}_{h}\right\}, \\
\Theta_{h}=W_{h}^{2} \oplus B_{h},
\end{gathered}
$$

where $B_{h}$ is the edge bubble space (see $[10,15]$ for more details). In that case, $\Gamma_{h}$ is chosen as the lowest order Nédélec finite element space, namely

$$
\Gamma_{h}=\left\{\sigma \in H_{0}(\text { rot }, \Omega) ; \sigma_{\mid T} \in \mathbb{P}_{0}(T)^{2} \oplus \mathbb{P}_{0}(T)\left(x_{2},-x_{1}\right)^{\top} \forall T \in \mathcal{T}_{h}\right\}
$$

and the reduction operator $\mathbf{R}_{h}$ is the associated interpolation operator that is characterized as follows: for any $\psi \in H_{0}($ rot,$\Omega), \mathbf{R}_{h} \psi$ is the unique element in $\Gamma_{h}$ satisfying

$$
\int_{E}\left(\mathbf{R}_{h} \psi-\psi\right) \cdot \tau_{E} d s=0
$$

for all edges $E$ of $T$ and any $T \in \mathcal{T}_{h}$. The advantage of this element is that it is locking free (see [15] for a robust a priori estimate). Other examples are also possible, we refer to Table 1 of [10] for a comprehensive list. In that case, our a posteriori error analysis is valid, but the robust a priori error analysis remains open for some of these elements (for instance, the MITC3 element).

By the usual Helmholtz decomposition of any $H_{0}($ rot,$\Omega)$ vector field [8, p. 299], for any $\phi_{h} \in \Theta_{h}$ there exist $z \in H_{0}^{1}(\Omega)$ and $\beta \in H_{0}^{1}(\Omega)^{2}$ such that,

$$
\left(\mathbf{R}_{h}-I\right) \phi_{h}=\nabla z-\beta
$$

as well as a constant $C>0$ such that

$$
\|z\|_{1}^{2}+\|\beta\|_{1}^{2} \leq C\left\|\left(\mathbf{R}_{h}-I\right) \phi_{h}\right\|_{H(r o t, \Omega)}^{2} .
$$

More precisely, if we introduce the constant $c_{R}$ such that

$$
|\beta|_{1} \leq c_{R}\left\|\operatorname{rot}\left(\mathbf{R}_{h}-I\right) \phi_{h}\right\|
$$

then we have $C=\left(1+c_{F}^{2}\right)\left(1+c_{R}^{2}+c_{R}^{2} c_{F}^{2}\right)$.

If $(\omega, \phi)$ is the solution of (2) and $\left(\omega_{h}, \phi_{h}\right)$ the one of $(4)$, the usual error $e_{h}^{e v}$ is defined as

$$
\begin{aligned}
\left(e_{h}^{e v}\right)^{2}=\left|\omega-\omega_{h}\right|_{1}^{2} & +\left|\phi-\phi_{h}\right|_{1}^{2}+\zeta^{-1} t^{2}\left\|\gamma-\gamma_{h}\right\|^{2} \\
& +\zeta^{-2} t^{4}\left\|\operatorname{rot}\left(\gamma-\gamma_{h}\right)\right\|^{2}+\left\|\gamma-\gamma_{h}\right\|_{-1}^{2}
\end{aligned}
$$


The residuals are defined as follows :

$$
\begin{aligned}
& \widehat{\operatorname{Res}_{1}}(v)=\left(\alpha_{t, h} \omega_{h}, v\right)-\left(\gamma_{h}, \nabla v\right) \quad \text { for any } v \in H_{0}^{1}(\Omega) \\
& \widehat{\operatorname{Res}_{2}}(\psi)=-a\left(\phi_{h}, \psi\right)+\left(\gamma_{h}, \psi\right)+\frac{t^{2}}{12}\left(\alpha_{t, h} \phi_{h}, \psi\right) \quad \text { for any } \psi \in H_{0}^{1}(\Omega)^{2} .
\end{aligned}
$$

We finally need to introduce the following mesh-dependent norm. For all $(\psi, v) \in$ $H_{0}^{1}(\Omega) \times H_{0}^{1}(\Omega)^{2}$, we define

$$
\left|\|(\psi, v) \mid\|_{1, h}^{2}=\|\nabla \psi\|^{2}+\sum_{T \in \mathcal{T}_{h}} \frac{1}{t^{2}+h_{T}^{2}}\|\nabla v-\psi\|_{T}^{2} .\right.
$$

For any functional $F$ defined on $H_{0}^{1}(\Omega) \times H_{0}^{1}(\Omega)^{2}$, the dual norm associated with $(11)$ is classically defined by

$$
\left.|\| F|\right|_{-1, h}=\sup _{(\psi, v) \in H_{0}^{1}(\Omega) \times H_{0}^{1}(\Omega)^{2} \backslash\{0\}} \frac{F(\psi, v)}{\left|\|(\psi, v) \mid\|_{1, h}\right.} .
$$

In the following, the notation $a \lesssim b$ and $a \sim b$ mean the existence of positive constants $c_{1}$ and $c_{2}$, which are independent of the mesh size, of the plate thickness parameter $t$, of the quantities $a$ and $b$ under consideration and of the coefficients of the operators such that $a \lesssim c_{2} b$ and $c_{1} b \lesssim a \lesssim c_{2} b$, respectively. The constants may in particular depend on the aspect ratio $\sigma$ of the mesh. We denote by $\overline{\omega_{T}}$ the union of elements $T^{\prime} \in \mathcal{T}_{h}$ that share at least a node with $T$ and by $\overline{\omega_{E}}$ the union of elements having in common the edge $E$. Finally, $\mathcal{E}_{h}$ denote the set of interiors edges in $\mathcal{T}_{h}$ and, for any edge $E \in \mathcal{E}_{h}$, we define by $h_{E}$ its length and by $n_{E}$ a fixed unit normal vector to $E$.

\section{Robust a priori estimations}

This section is devoted to an a priori error analysis of the Reissner-Mindlin eigenvalue problem. This subject is the origin of a lot of works (see e.g. [16], [17], [18], [22], [25], [31]) in the smooth case, in the sense that the domain is supposed to have a smooth boundary or to be a convex polygon. Here we want to perform a similar analysis without the convexity assumption. This requires to revisit the whole results with less regular solutions. We first start with robust a priori estimates for the Reissner-Mindlin system with data in $L^{2}(\Omega)$ and then give their consequence to the eigenvalue problem.

\subsection{Robust a priori estimates for the Reissner-Mindlin system}

As suggested before, we need to determine the regularity properties and to give uniform estimates of the solution of the Reissner-Mindlin system with $L^{2}$ right-hand side. For this purpose, let us consider the following problem : Given $g \in L^{2}(\Omega)$ and $\varphi \in L^{2}(\Omega)^{2}$, find 
$\left(\beta_{t}, w_{t}\right) \in H_{0}^{1}(\Omega)^{2} \times H_{0}^{1}(\Omega)$ such that for all $(\eta, v) \in H_{0}^{1}(\Omega)^{2} \times H_{0}^{1}(\Omega)$

$$
\left\{\begin{array}{l}
a\left(\beta_{t}, \eta\right)+\left(\tau_{t}, \nabla v-\eta\right)=(g, v)+\frac{t^{2}}{12}(\varphi, \eta) \\
\tau_{t}=\zeta t^{-2}\left(\nabla w_{t}-\beta_{t}\right) .
\end{array}\right.
$$

This problem has a unique solution in $H_{0}^{1}(\Omega)^{2} \times H_{0}^{1}(\Omega)$ since the bilinear form

$$
((\beta, w),(\eta, v)) \rightarrow a(\beta, \eta)+\zeta t^{-2}(\nabla w-\beta, \nabla v-\eta)
$$

is coercive in $H_{0}^{1}(\Omega)^{2} \times H_{0}^{1}(\Omega)$.

For such a problem we have the following regularity result with robust a priori estimates (in the regular case, see Theorem 7.1 of [2]).

Theorem 2.1 There exists $\varepsilon_{0} \in\left(0, \frac{1}{2}\right]$ such that for all $\varepsilon \in\left(0, \varepsilon_{0}\right],\left(\beta_{t}, w_{t}\right) \in H^{3 / 2+\varepsilon}(\Omega)^{2} \times$ $H^{3 / 2+\varepsilon}(\Omega)$ with

$$
\left\|\beta_{t}\right\|_{3 / 2+\varepsilon}+\left\|w_{t}\right\|_{3 / 2+\varepsilon}+\left\|\tau_{t}\right\|_{-1 / 2+\varepsilon}+t\left\|\tau_{t}\right\|_{1 / 2+\varepsilon} \lesssim\|g\|+t^{2}\|\varphi\|
$$

Proof: As in [2], we see that $\left(\beta_{t}, w_{t}\right) \in H_{0}^{1}(\Omega)^{2} \times H_{0}^{1}(\Omega)$ is the unique solution of (13) if and only if $\left(r, \beta_{t}, p_{t}, w_{t}\right) \in H_{0}^{1}(\Omega) \times H_{0}^{1}(\Omega)^{2} \times \hat{H}^{1}(\Omega) \times H_{0}^{1}(\Omega)$ is solution of the triangular system

$$
\left\{\begin{array}{l}
\zeta(\nabla r, \nabla \mu)=(g, \mu), \forall \mu \in H_{0}^{1}(\Omega), \\
a\left(\beta_{t}, \psi\right)-\zeta\left(\operatorname{curl} p_{t}, \psi\right)=\zeta(\nabla r, \psi)+\frac{t^{2}}{12}(\varphi, \psi), \forall \psi \in H_{0}^{1}(\Omega)^{2}, \\
-\left(\beta_{t}, \operatorname{curl} q\right)-t^{2}\left(\operatorname{curl} p_{t}, \operatorname{curl} q\right)=0, \forall q \in \hat{H}^{1}(\Omega) \\
\left(\nabla w_{t}, \nabla s\right)=\left(\beta_{t}+t^{2} \nabla r_{t}, \nabla s\right), \forall s \in H_{0}^{1}(\Omega),
\end{array}\right.
$$

with the relation

$$
t^{-2}\left(\nabla w_{t}-\beta_{t}\right)=\nabla r+\operatorname{curl} p_{t}
$$

and the notation

$$
\hat{H}^{1}(\Omega)=H^{1}(\Omega) \cap \hat{L}^{2}(\Omega), \quad \hat{L}^{2}(\Omega)=\left\{q \in L^{2}(\Omega): \int_{\Omega} q(x) d x=0\right\} .
$$

Now we divide the proof is different steps:

1) The first problem in (15) is a Dirichlet problem in $\Omega$ with a $L^{2}(\Omega)$ datum, therefore by [24, Corollary 2.4.4], there exists $\varepsilon_{\Delta} \in\left(0, \frac{1}{2}\right]$ such that $r \in H^{3 / 2+\varepsilon}(\Omega)$, for all $\varepsilon \in\left(0, \varepsilon_{\Delta}\right]$ and

$$
\|r\|_{3 / 2+\varepsilon} \lesssim\|g\|
$$

2) We now look at the system in $\left(\beta_{t}, p_{t}\right)$ that by taking the difference between the second and the third line of (15) (multiplied by $\zeta$ ) takes the form

$a\left(\beta_{t}, \psi\right)-\zeta\left(\operatorname{curl} p_{t}, \psi\right)+\zeta\left(\beta_{t}, \operatorname{curl} q\right)+\zeta t^{2}\left(\operatorname{curl} p_{t}, \operatorname{curl} q\right)=\langle F, \psi\rangle, \forall(\psi, q) \in H_{0}^{1}(\Omega)^{2} \times \hat{H}^{1}(\Omega)$, 
where here $F:=\zeta \nabla r+\frac{t^{2}}{12} \varphi$. Again this problem has a unique solution for any $F \in H^{-1}(\Omega)^{2}$ since the left-hand side is coercive in $H_{0}^{1}(\Omega)^{2} \times \hat{H}^{1}(\Omega)$. By taking $(\psi, q)=\left(\beta_{t}, p_{t}\right)$ and using Korn's inequality, we get

$$
\left\|\beta_{t}\right\|_{1}^{2}+t^{2}\left\|\operatorname{curl} p_{t}\right\|^{2} \lesssim\|F\|_{-1}\left\|\beta_{t}\right\|_{1}
$$

and therefore

$$
\left\|\beta_{t}\right\|_{1}+t\left\|\operatorname{curl} p_{t}\right\| \lesssim\|F\|_{-1} .
$$

But by taking $\psi=0$ in (17), we get

$$
\left(\beta_{t}, \operatorname{curl} q\right)+t^{2}\left(\operatorname{curl} p_{t}, \operatorname{curl} q\right)=0, \forall q \in H^{1}(\Omega)
$$

since the curl of a constant function is zero. By integration by parts, we get equivalently

$$
\left(\operatorname{curl} \beta_{t}, q\right)=-t^{2}\left(\operatorname{curl} p_{t}, \operatorname{curl} q\right), \forall q \in H^{1}(\Omega) .
$$

By Cauchy-Schwarz's inequality in the right-hand side, we obtain

$$
\left\|\operatorname{curl} \beta_{t}\right\|_{-1}=\sup _{q \in H_{0}^{1}(\Omega), q \neq 0} \frac{\left|\left(\operatorname{curl} \beta_{t}, q\right)\right|}{\|q\|_{1}} \lesssim t^{2}\left\|\operatorname{curl} p_{t}\right\|,
$$

and by (18) we arrive at

$$
\left\|\operatorname{curl} \beta_{t}\right\|_{-1} \lesssim t\|F\|_{-1}
$$

We now look at an estimate of the $L^{2}$-norm of $p_{t}$. For that purpose, we notice that by Corollary I.2.4 of [21] there exists $\phi^{0} \in H_{0}^{1}(\Omega)^{2}$ such that

$$
\operatorname{div} \phi^{0}=p_{t} \quad \text { in } \Omega,
$$

and

$$
\left\|\phi^{0}\right\|_{1} \lesssim\left\|p_{t}\right\|
$$

Therefore the function $\psi^{0}=\left(-\phi_{2}^{0}, \phi_{1}^{0}\right)$ belongs to $H_{0}^{1}(\Omega)^{2}$ and satisfies

$$
\operatorname{curl} \psi^{0}=\operatorname{div} \phi^{0}=p_{t} \quad \text { in } \Omega \text {, }
$$

as well as

$$
\left\|\psi^{0}\right\|_{1} \lesssim\left\|p_{t}\right\|
$$

Using as test function in (17) the pair $\left(\psi^{0}, 0\right)$, we get

$$
\zeta\left(\operatorname{curl} p_{t}, \psi^{0}\right)=a\left(\beta_{t}, \psi^{0}\right)-\left\langle F, \psi^{0}\right\rangle .
$$

Using (21) and Green's formula, we obtain

$$
\zeta\left\|p_{t}\right\|^{2}=\zeta\left(p_{t}, \operatorname{curl} \psi^{0}\right)=\zeta\left(\operatorname{curl} p_{t}, \psi^{0}\right)=a\left(\beta_{t}, \psi^{0}\right)-\left\langle F, \psi^{0}\right\rangle .
$$


Using Cauchy-Schwarz's inequality, (18) and (22), we arrive at

$$
\left\|p_{t}\right\| \lesssim\|F\|_{-1} .
$$

Let us now introduce the mapping $\mathcal{A}_{0}$ as follows

$$
\mathcal{A}_{0}: H^{-1}(\Omega)^{2} \rightarrow H_{0}^{1}(\Omega)^{2} \times \hat{L}^{2}(\Omega): F \rightarrow\left(\beta_{0}, p_{0}\right),
$$

where $\left(\beta_{0}, p_{0}\right) \in H_{0}^{1}(\Omega)^{2} \times \hat{L}^{2}(\Omega)$ is the unique solution of the Stokes like system (that formally corresponds to (17) with $t=0$ )

$$
\left\{\begin{array}{l}
a\left(\beta_{0}, \psi\right)-\zeta\left(p_{0}, \operatorname{curl} \psi\right)=\langle F, \psi\rangle, \forall \psi \in H_{0}^{1}(\Omega)^{2} \\
\left(\operatorname{curl} \beta_{0}, q\right)=0, \forall q \in \hat{L}^{2}(\Omega) .
\end{array}\right.
$$

Clearly (see $\left[2\right.$, p. 1288]) $\mathcal{A}_{0}$ is an isomorphism and consequently for all $t \in\left(0, t_{\text {max }}\right]$ we can consider the mapping

$$
B_{t}: H_{0}^{1}(\Omega)^{2} \times \hat{L}^{2}(\Omega) \rightarrow \hat{L}^{2}(\Omega) \times H^{-1}(\Omega):\left(\beta_{0}, p_{0}\right) \rightarrow\left(p_{t}, \operatorname{curl} \beta_{t}\right)
$$

where $\left(\beta_{t}, p_{t}\right)$ is the unique solution of (17) with right-hand side $F=\mathcal{A}_{0}^{-1}\left(\beta_{0}, p_{0}\right)$.

First we notice that the estimates (20) and (23) imply that $B_{t}$ is uniformly (in $t$ ) bounded in the sense that

$$
\left\|p_{t}\right\|+t^{-1}\left\|\operatorname{curl} \beta_{t}\right\|_{-1} \lesssim\left\|\beta_{0}\right\|_{1}+\left\|p_{0}\right\|
$$

On the other hand the proof of Theorem 7.1 of [2] shows that $B_{t}$ is also uniformly bounded from $H^{2}(\Omega)^{2} \cap H_{0}^{1}(\Omega)^{2} \times \hat{H}^{1}(\Omega)$ to $\hat{H}^{1}(\Omega) \times L^{2}(\Omega)$ in the sense that

$$
\|p\|_{1}+t^{-1}\left\|\operatorname{curl} \beta_{t}\right\|_{0} \lesssim\left\|\beta_{0}\right\|_{2}+\left\|p_{0}\right\|_{1}
$$

reminding that $\operatorname{curl} \beta_{0}=0$.

Therefore by interpolation, the mapping $B_{t}$ is uniformly bounded from $H^{1+s}(\Omega)^{2} \cap$ $H_{0}^{1}(\Omega)^{2} \times \hat{H}^{s}(\Omega)$ to $\hat{H}^{s}(\Omega) \times H^{s-1}(\Omega)$, for all $s \in[0,1], s \neq 1 / 2$ (for $s=1 / 2$, the statement is also valid but the target space should be changed into $\left.\hat{H}^{1 / 2}(\Omega) \times\left(\tilde{H}^{1 / 2}(\Omega)\right)^{\prime}\right)$ with the estimate

$$
\|p\|_{s}+t^{-1}\left\|\operatorname{curl} \beta_{t}\right\|_{s-1} \lesssim\left\|\beta_{0}\right\|_{1+s}+\left\|p_{0}\right\|_{s} .
$$

Let us show that this implies that there exists $\varepsilon_{0} \in\left(0, \frac{1}{2}\right]$ such that for all $\varepsilon \in\left(0, \varepsilon_{0}\right]$ $\left(\beta_{t}, p_{t}\right)$ belongs to $H^{3 / 2+\varepsilon}(\Omega)^{2} \times H^{3 / 2+\varepsilon}(\Omega)$ with the estimate

$$
\left\|\beta_{t}\right\|_{3 / 2+\varepsilon}+\left\|p_{t}\right\|_{1 / 2+\varepsilon}+t\|p\|_{3 / 2+\varepsilon} \lesssim\|F\|_{-1 / 2+\varepsilon} .
$$

Indeed by [24, Theorem 6.2.3] and [34, section 6.2], there exists $\varepsilon_{S} \in\left(0, \frac{1}{2}\right]$ such that for all $\varepsilon \in\left(0, \varepsilon_{S}\right], \mathcal{A}_{0}$ is an isomorphism from $H^{-1 / 2+\varepsilon}(\Omega)$ into $H^{3 / 2+\varepsilon}(\Omega)^{2} \cap H_{0}^{1}(\Omega)^{2} \times$ $H^{1 / 2+\varepsilon}(\Omega) \cap \hat{L}^{2}(\Omega)$. Hence by the property $(25)$ of $B_{t}$ with $s=1 / 2+\varepsilon$, we get

$$
\left\|p_{t}\right\|_{1 / 2+\varepsilon}+t^{-1}\left\|\operatorname{curl} \beta_{t}\right\|_{-1 / 2+\varepsilon} \lesssim\|F\|_{-1 / 2+\varepsilon} .
$$


At this stage, we can look at $\beta_{t} \in H_{0}^{1}(\Omega)^{2}$ solution of the elasticity system

$$
a\left(\beta_{t}, \psi\right)=\left\langle F+\zeta \operatorname{curl} p_{t}, \psi\right\rangle \forall \psi \in H_{0}^{1}(\Omega)^{2}
$$

and using [23, Thm 6.1] and [34, section 6.1], there exists $\varepsilon_{L} \in\left(0, \frac{1}{2}\right]$ such that for all $\varepsilon \in\left(0, \varepsilon_{L}\right], \beta_{t} \in H^{3 / 2+\varepsilon}(\Omega)^{2}$ if $F+\zeta \operatorname{curl} p_{t} \in H^{-1 / 2+\varepsilon}(\Omega)^{2}$ with the estimate

$$
\left\|\beta_{t}\right\|_{3 / 2+\varepsilon} \lesssim\left\|F+\zeta \operatorname{curl} p_{t}\right\|_{-1 / 2+\varepsilon}
$$

In a second step, as (19) means that $p_{t} \in \hat{H}^{1}(\Omega)$ is the unique solution of the Neumann problem

$$
\begin{cases}\Delta p_{t}=t^{-2} \operatorname{curl} \beta_{t} & \text { in } \Omega \\ \partial_{n} p_{t}=0 & \text { on } \partial \Omega .\end{cases}
$$

Hence if $\varepsilon \in\left(0, \varepsilon_{\Delta}\right]$ by $[13, ?]$, we find that $p_{t}$ belongs to $H^{3 / 2+\varepsilon}(\Omega)$ with the estimate

$$
\left\|p_{t}\right\|_{3 / 2+\varepsilon} \lesssim t^{-2}\left\|\operatorname{curl} \beta_{t}\right\|_{-1 / 2+\varepsilon}
$$

Consequently for $\varepsilon_{0} \leq \min \left\{\varepsilon_{\Delta}, \varepsilon_{S}\right\}$, by $(27)$, we get

$$
t\left\|p_{t}\right\|_{3 / 2+\varepsilon} \lesssim\|F\|_{-1 / 2+\varepsilon}
$$

The estimate (26) then follows from (27), (28) and (29) by choosing $\varepsilon_{0}=\min \left\{\varepsilon_{S}, \varepsilon_{L}, \varepsilon_{\Delta}\right\}$.

Coming back to problem (15), the right-hand side of (17) is given by $F:=\zeta \nabla r+\frac{t^{2}}{12} \varphi$. Hence by (16) and (26), for all $\varepsilon \in\left(0, \varepsilon_{f}\right],\left(\beta_{t}, p_{t}\right)$ belongs to $H^{3 / 2+\varepsilon}(\Omega)^{2} \times H^{3 / 2+\varepsilon}(\Omega)$ with the estimate

$$
\left\|\beta_{t}\right\|_{3 / 2+\varepsilon}+\left\|p_{t}\right\|_{1 / 2+\varepsilon}+t\|p\|_{3 / 2+\varepsilon} \lesssim\|g\|+t^{2}\|\varphi\|
$$
of

3) The last identity in (15) means that $w_{t} \in H_{0}^{1}(\Omega)$ can be seen as the unique solution

$$
\left(\nabla w_{t}, \nabla s\right)=\left(\beta_{t}+t^{2} \nabla r_{t}, \nabla s\right), \forall s \in H_{0}^{1}(\Omega)
$$

Hence for all $\varepsilon \in\left(0, \varepsilon_{f}\right], w_{t}$ belongs to $H^{3 / 2+\varepsilon}(\Omega)$ with the estimate

$$
\left\|w_{t}\right\|_{3 / 2+\varepsilon} \lesssim\left\|\beta_{t}+t^{2} \nabla r_{t}\right\|_{-1 / 2+\varepsilon}
$$

Combined with (16) and (30) we have obtained

$$
\left\|w_{t}\right\|_{3 / 2+\varepsilon} \lesssim\|g\|+t^{2}\|\varphi\|
$$

Finally recalling that $\tau_{t}=\zeta t^{-2}\left(\nabla w_{t}-\beta_{t}\right)=\zeta\left(\nabla r+\operatorname{curl} p_{t}\right)$, the estimate (14) is a simple consequence of (16), (30) and (31). 


\subsection{Robust a priori error estimates for the eigenvalue problem}

In order to perform the error analysis between the exact eigenvalues of (2) and their approximation (eigenvalues of (4)), it is convenient to introduce the operator

$$
T_{t}: L^{2}(\Omega)^{2} \times L^{2}(\Omega) \rightarrow L^{2}(\Omega)^{2} \times L^{2}(\Omega):(\varphi, g) \rightarrow T_{t}(\varphi, g)=\left(\beta_{t}, w_{t}\right),
$$

where $\left(\beta_{t}, w_{t}\right) \in H_{0}^{1}(\Omega)^{2} \times H_{0}^{1}(\Omega)$ is the unique solution of $(13)$ with datum $(\varphi, g)$. As the bilinear form $a$ introduced before is symmetric, $T_{t}$ is a selfadjoint and compact operator from $L^{2}(\Omega)^{2} \times L^{2}(\Omega)$ into itself equipped with the natural inner product and norm

$$
|(\varphi, g)|_{t}^{2}=\frac{t^{2}}{12}\|\varphi\|^{2}+\|g\|^{2}
$$

Furthermore $\alpha_{t}$ is an eigenvalue of (2) if and only if $\frac{1}{\alpha_{t}}$ is an eigenvalue of $T_{t}$.

As $t \rightarrow 0$ (cfr. [8]), the solution $\left(\beta_{t}, w_{t}\right)$ of (13) converges to $\left(\beta_{0}, w_{0}\right) \in H_{0}^{1}(\Omega)^{2} \times H_{0}^{1}(\Omega)$, where $\left(\beta_{0}, p_{0}\right)$ is the unique solution of $(24), w_{0} \in H_{0}^{2}(\Omega)$ is the unique solution of

$$
\frac{1}{12(1+\nu)} \Delta^{2} w_{0}=f \text { in } \Omega
$$

Setting $\tau_{0}=\zeta\left(\nabla r+\operatorname{curl} p_{0}\right)$ (that belongs to $\left.H_{0}(\operatorname{curl}, \Omega)^{\prime}\right)$, it holds

$$
\left\{\begin{array}{l}
a\left(\beta_{0}, \psi\right)+\left\langle\tau_{0}, \nabla v-\psi\right\rangle=(g, v), \forall(\eta, v) \in H_{0}^{1}(\Omega)^{2} \times H_{0}^{1}(\Omega), \\
\beta_{0}=\nabla w_{0} .
\end{array}\right.
$$

Let us notice that the regularity results from Theorem 2.1 only yield $\tau_{0} \in H^{-1 / 2+\varepsilon}(\Omega)$ for some $\varepsilon \in(0,1 / 2]$.

As before we define the operator $T_{0}$ by

$$
T_{0}: L^{2}(\Omega)^{2} \times L^{2}(\Omega) \rightarrow L^{2}(\Omega)^{2} \times L^{2}(\Omega):(\varphi, g) \rightarrow T_{0}(\varphi, g)=\left(\beta_{0}, w_{0}\right) .
$$

The first aim is to prove that $T_{t}$ tends to $T_{0}$ as $t$ goes to zero even in the non-convex case (see Lemma 3.1 of [17] in the convex case):

Lemma 2.2 For all $(\varphi, g) \in L^{2}(\Omega)^{2} \times L^{2}(\Omega)$, it holds

$$
\left\|\left(T_{t}-T_{0}\right)(\varphi, g)\right\|_{H_{0}^{1}(\Omega)^{2} \times H_{0}^{1}(\Omega)} \lesssim \sqrt{t}|(\varphi, g)|_{t}
$$

Proof: Subtracting (32) to (13) we have

$$
a\left(\beta_{t}-\beta_{0}, \psi\right)+\left\langle\tau_{t}-\tau_{0}, \nabla v-\psi\right\rangle=\frac{t^{2}}{12}(\varphi, \eta), \forall(\eta, v) \in H_{0}^{1}(\Omega)^{2} \times H_{0}^{1}(\Omega) .
$$

Hence taking $\eta=\beta_{t}-\beta_{0}$ and $v=w_{t}-w_{0}$, we find

$$
a\left(\beta_{t}-\beta_{0}, \beta_{t}-\beta_{0}\right)=\frac{t^{2}}{12}\left(\varphi, \beta_{t}-\beta_{0}\right)-\frac{t^{2}}{\zeta}\left\langle\tau_{t}-\tau_{0}, \tau_{t}\right\rangle
$$


Using the (uniform) coerciveness of $a$, Cauchy-Schwarz's inequality and the a priori estimate (14), we get

$$
\begin{aligned}
\left\|\beta_{t}-\beta_{0}\right\|_{1}^{2} & \leq \frac{t^{2}}{12}\|\varphi\|\left\|\beta_{t}-\beta_{0}\right\|+\frac{t^{2}}{\zeta}\left\|\tau_{t}-\tau_{0}\right\|_{-1 / 2+\varepsilon}\|\| \tau_{t} \|_{1 / 2-\varepsilon} \\
& \lesssim t|(\varphi, g)|_{t}\left\|\beta_{t}-\beta_{0}\right\|_{1}+t|(\varphi, g)|_{t}^{2} .
\end{aligned}
$$

Hence Young's inequality leads to

$$
\left\|\beta_{t}-\beta_{0}\right\|_{1} \lesssim \sqrt{t}|(\varphi, g)|_{t} .
$$

Observing that

$$
\nabla\left(w_{t}-w_{0}\right)=\beta_{t}-\beta_{0}+\frac{t^{2}}{\zeta} \tau_{t}
$$

we get

$$
\begin{aligned}
\left\|\nabla\left(w_{t}-w_{0}\right)\right\| & \leq\left\|\beta_{t}-\beta_{0}\right\|+\frac{t^{2}}{\zeta}\left\|\tau_{t}\right\| \\
& \lesssim\left\|\beta_{t}-\beta_{0}\right\|+t^{2}\left\|\tau_{t}\right\|_{1 / 2+\varepsilon}
\end{aligned}
$$

The conclusion then follows from the previous estimate (33) and (14).

Once such a convergence result is obtained by standard perturbation arguments (see for instance [28] and [17] for its application to the Reissner-Mindlin system), we obtain the next result.

Lemma 2.3 Let $\mu_{0}>0$ be a fixed eigenvalue of $T_{0}$ of algebraic multiplicity $m$ and let $D$ be a open disc of the complex plane centred at $\mu_{0}$ that contains no other element of the spectrum of $T_{0}$. Then there exists $t_{0}>0$ (depending on $\mu_{0}$ ) such that for all $t \in\left(0, t_{0}\right], T_{t}$ contains exactly $m$ eigenvalues in $D$ (repeated according to their algebraic multiplicities). In particular $\mu_{0}$ is the limit of eigenvalues of $T_{t}$. Furthermore if $\mu_{0}$ is a simple eigenvalue of $T_{0}$, then $T_{t}$ has a simple eigenvalue $\mu_{t}$ in $D$ for all $t \leq t_{0}$ and the distance of $\mu_{t}$ to the remainder of the spectrum of $T_{t}$ remains uniformly bounded from below.

We are now ready to prove some convergence results between exact eigenvalues and eigenvectors and discrete ones:

Theorem 2.4 Let $\mu_{0}>0$ be a simple eigenvalue of $T_{0}$ and fix $t_{0}$ small enough such that $T_{t}$ fulfils the properties of Lemma 2.3, in particular denote by $\mu_{t}$ its eigenvalue that converges to $\mu_{0}$. Let $\alpha_{t}=\frac{1}{\mu_{t}}$ that is a simple eigenvalue of problem (2) and let $(\omega, \phi) \in$ $H_{0}^{1}(\Omega)^{2} \times H_{0}^{1}(\Omega)$ be its corresponding normalized eigenvector, i.e., $|(\omega, \phi)|_{t}=1$. Then there exist $h_{0}>0$, and $\varepsilon \in\left(0, \frac{1}{2}\right]$ such that for all $h<h_{0}$, the discrete problem (4) has a unique eigenvalue $\alpha_{t, h}$ that converges to $\alpha_{t}$ as $h$ goes to zero. Furthermore if $\left(\omega_{h}, \phi_{h}\right) \in W_{h} \times \Theta_{h}$ is the corresponding normalized eigenvector, i.e., $\left|\left(\omega_{h}, \phi_{h}\right)\right|_{t}=1$, then one has

$$
\begin{aligned}
\left\|\phi-\phi_{h}\right\|_{1}+\left\|\omega-\omega_{h}\right\|_{1} & \lesssim h^{1 / 2+\varepsilon} \\
\left\|\phi-\phi_{h}\right\|+\left\|\omega-\omega_{h}\right\| & \lesssim h^{1+2 \varepsilon} \\
\left|\alpha_{t}-\alpha_{t, h}\right| & \lesssim h^{1+2 \varepsilon} .
\end{aligned}
$$


Proof: Given $(\varphi, g) \in L^{2}(\Omega)^{2} \times L^{2}(\Omega)$, we consider $\left(\beta_{t, h}, w_{t, h}\right) \in W_{h} \times \Theta_{h}$ solution of

$$
\left\{\begin{array}{l}
a\left(\beta_{t, h}, \eta_{h}\right)+\left(\tau_{t, h}, \nabla v_{h}-\mathbf{R}_{h} \eta_{h}\right)=\left(g, v_{h}\right)+\frac{t^{2}}{12}\left(\varphi, \eta_{h}\right), \forall\left(v_{h}, \eta_{h}\right) \in W_{h} \times \Theta_{h} \\
\tau_{t, h}=\zeta t^{-2}\left(\nabla w_{t, h}-\mathbf{R}_{h} \beta_{t, h}\right) .
\end{array}\right.
$$

and the mapping

$$
T_{t, h}: L^{2}(\Omega)^{2} \times L^{2}(\Omega) \rightarrow L^{2}(\Omega)^{2} \times L^{2}(\Omega):(\varphi, g) \rightarrow T_{t, h}(\varphi, g)=\left(\beta_{t, h}, w_{t, h}\right) .
$$

As in Lemma 3.2 of [17], we prove that for all $(\varphi, g) \in L^{2}(\Omega)^{2} \times L^{2}(\Omega)$, it holds

$$
\left\|\left(T_{t}-T_{t, h}\right)(\varphi, g)\right\|_{H_{0}^{1}(\Omega)^{2} \times H_{0}^{1}(\Omega)} \lesssim h^{1 / 2+\varepsilon}|(\varphi, g)|_{t}
$$

Indeed the only difference is to use the estimate

$$
\left\|\beta_{t}-\beta_{t, h}\right\|_{1}+t\left\|\tau_{t}-\tau_{t, h}\right\| \lesssim h^{1 / 2+\varepsilon}\left(\left\|\beta_{t}\right\|_{3 / 2+\varepsilon}+t\left\|\tau_{t}\right\|_{1 / 2+\varepsilon}+\left\|\tau_{t}\right\|_{-1 / 2+\varepsilon}\right) .
$$

If this estimate holds then by (14) we will get

$$
\left\|\beta_{t}-\beta_{t, h}\right\|_{1}+t\left\|\tau_{t}-\tau_{t, h}\right\| \lesssim h^{1 / 2+\varepsilon}\left(\|g\|+t^{2}\|\varphi\|\right),
$$

and consequently

$$
\left\|w_{t}-w_{t, h}\right\|_{1} \lesssim h^{1 / 2+\varepsilon}\left(\|g\|+t^{2}\|\varphi\|\right),
$$

since

$$
\begin{aligned}
\nabla\left(w_{t}-w_{t, h}\right) & =\beta_{t}-\mathbf{R}_{h} \beta_{t, h}+\frac{t^{2}}{\zeta}\left(\tau_{t}-\tau_{t, h}\right) \\
& =\beta_{t}-\mathbf{R}_{h} \beta_{t}+\mathbf{R}_{h}\left(\beta_{t}-\beta_{t, h}\right)+\frac{t^{2}}{\zeta}\left(\tau_{t}-\tau_{t, h}\right) .
\end{aligned}
$$

Hence using standard propertries of $\mathbf{R}_{h}$ we get

$$
\begin{aligned}
\| \nabla\left(w_{t}-w_{t, h}\right) & \leq\left\|\beta_{t}-\mathbf{R}_{h} \beta_{t}\right\|+\left\|\mathbf{R}_{h}\left(\beta_{t}-\beta_{t, h}\right)\right\|+\frac{t^{2}}{\zeta}\left\|\tau_{t}-\tau_{t, h}\right\| \\
& \lesssim h\left\|\beta_{t}\right\|_{1}+\left\|\beta_{t}-\beta_{t, h}\right\|_{1}+t\left\|\tau_{t}-\tau_{t, h}\right\|,
\end{aligned}
$$

which yields (39) thanks to (38) and (14).

To prove (38) we adapt Lemma 3.1 of [15] to our setting by proving that for any $(\hat{\beta}, \hat{w}) \in W_{h} \times \Theta_{h}$, setting $\hat{\tau}=\zeta t^{-2}\left(\nabla \hat{w}-\mathbf{R}_{h} \hat{\beta}\right)$, we have

$$
\left\|\hat{\beta}-\beta_{t, h}\right\|_{1}+t\left\|\hat{\tau}-\tau_{t, h}\right\|_{1} \lesssim\left\|\hat{\beta}-\beta_{t}\right\|_{1}+t\left\|\hat{\tau}-\tau_{t}\right\|+h^{1 / 2+\varepsilon}\left\|\gamma_{t}\right\|_{-1 / 2+\varepsilon}
$$

Indeed as in Lemma 3.1 of [15], we may write

$$
\begin{aligned}
a\left(\hat{\beta}-\beta_{t, h}, \hat{\beta}-\beta_{t, h}\right)+\frac{t^{2}}{\zeta}\left(\hat{\tau}-\tau_{t, h}, \hat{\tau}-\tau_{t, h}\right) & =a\left(\hat{\beta}-\beta_{t}, \hat{\beta}-\beta_{t, h}\right)+\frac{t^{2}}{\zeta}\left(\hat{\tau}-\tau_{t}, \hat{\tau}-\tau_{t, h}\right) \\
& +\left(\gamma_{t}, \hat{\beta}-\beta_{t, h}-\mathbf{R}_{h}\left(\hat{\beta}-\beta_{t, h}\right) .\right.
\end{aligned}
$$


Hence by the uniform coerciveness of $a$, Young's inequality and Cauchy-Schwarz's inequality we get

$$
\begin{aligned}
\left\|\hat{\beta}-\beta_{t, h}\right\|_{1}^{2}+t^{2}\left\|\hat{\tau}-\tau_{t, h}\right\|^{2} & \lesssim\left\|\hat{\beta}-\beta_{t}\right\|_{1}^{2}+t^{2}\left\|\hat{\tau}-\tau_{t}\right\|^{2} \\
& +\left\|\gamma_{t}\right\|_{-1 / 2+\varepsilon}\left\|\hat{\beta}-\beta_{t, h}-\mathbf{R}_{h}\left(\hat{\beta}-\beta_{t, h}\right)\right\|_{1 / 2-\varepsilon} .
\end{aligned}
$$

Hence using the estimate

$$
\left\|\eta-\mathbf{R}_{h} \eta\right\|_{1 / 2-\varepsilon} \lesssim h^{1 / 2+\varepsilon}\|\eta\|_{1},
$$

again by Young's inequality we arrive at (40).

This estimate (40), (14) and the arguments of Corollary 3.2 of [15] lead to (38).

The estimate (37) and Theorem 7.1 of [3] lead to (34) due to Lemma 2.3.

Since $\beta_{t}$ belongs to $H_{0}^{1}(\Omega)^{2}$, we can use the same duality argument than the one from Lemma 3.4 of [17] thanks to Lemma 3.3 of [17] and get

$$
\left\|\beta_{t}-\beta_{t, h}\right\|+\left\|w_{t}-w_{t, h}\right\| \lesssim h^{1+2 \varepsilon}\left(\|g\|+t^{2}\|\varphi\|\right) .
$$

In other words, for all $(\varphi, g) \in L^{2}(\Omega)^{2} \times L^{2}(\Omega)$, it holds

$$
\left\|\left(T_{t}-T_{t, h}\right)(\varphi, g)\right\|_{L^{2}(\Omega)^{2} \times L^{2}(\Omega)} \lesssim h^{1+2 \varepsilon}|(\varphi, g)|_{t} .
$$

and (35) follows as before.

In order to prove the relation (36), we use the same argument than in Theorem 2.2 of [17], namely applying Remark 7.5 of [3], we have

$$
\left|\mu_{t}-\mu_{t, h}\right| \leq C\left(\left|\left(T_{t}-T_{t, h}\right)\left(\beta_{t}, w_{t}\right)\right|_{t}+\left|\left(T_{t}-T_{t, h}\right)\left(\beta_{t}, w_{t}\right)\right|_{t}^{2}\right),
$$

where $C$ is a positive constant depending on the inverse of the distance from $\mu_{t}$ to the remainder of the spectrum of $T_{t}$. Hence by Lemma 2.3 and (42) we obtain

$$
\left|\mu_{t}-\mu_{t, h}\right| \lesssim h^{1+2 \varepsilon}
$$

As $\alpha_{t}=\frac{1}{\mu_{t}}$ and $\alpha_{t, h}=\frac{1}{\mu_{t, h}}$, we arrive at (36).

Remark 2.5 If $\Omega$ is convex, then we can take $\varepsilon=1 / 2$, and we recover standard results presented in most existing works (e.g. [16], [17], [18], [22], [25], [31]).

\section{Preliminary results}

The aim of this section is to prove three lemmas which will be used in the following of the paper. The proofs of Lemmas 3.2 and 3.3 are close (but non identical) to the ones of [10] and [12]. Nevertheless, we give them for the sake of completeness. 
Lemma 3.1 We have

$$
\left\|\gamma-\gamma_{h}\right\|_{-1}^{2} \leq 6(\mu+\lambda)\left\|\phi-\phi_{h}\right\|_{\mathcal{C}}^{2}+3\left\|\widehat{R e s_{2}}\right\|_{-1}^{2}+3 c_{F}^{2}\left(\frac{t^{2}}{12}\left\|\alpha_{t} \phi-\alpha_{t, h} \phi_{h}\right\|\right)^{2} .
$$

Proof: First, it can be shown that for any $\psi \in\left(H_{0}^{1}(\Omega)\right)^{2}(\mathrm{cf}[12])$,

$$
\|\psi\|_{\mathcal{C}}^{2} \leq 2(\mu+\lambda)|\psi|_{1}^{2}
$$

hence by (2), (4) and the definition of $\widehat{\operatorname{Res}_{2}}$, we get

$$
\begin{aligned}
\left(\gamma-\gamma_{h}, \psi\right) & =a(\phi, \psi)-\alpha_{t} \frac{t^{2}}{12}(\phi, \psi)-\left(\gamma_{h}, \psi\right) \\
& =a\left(\phi-\phi_{h}, \psi\right)-\widehat{\operatorname{Res}_{2}}(\psi)-\frac{t^{2}}{12}\left(\alpha_{t} \phi-\alpha_{t, h} \phi_{h}, \psi\right) \\
& \leq\left\|\phi-\phi_{h}\right\|_{\mathcal{C}}\|\psi\|_{\mathcal{C}}+\left\|\widehat{R e s_{2}}\right\|_{-1}|\psi|_{1}+\frac{t^{2}}{12}\left\|\alpha_{t} \phi-\alpha_{t, h} \phi_{h}\right\|\|\psi\| \\
& \leq\left((2(\mu+\lambda))^{1 / 2}\left\|\phi-\phi_{h}\right\|_{\mathcal{C}}+\left\|\widehat{R e s_{2}}\right\|_{-1}+c_{F} \frac{t^{2}}{12}\left\|\alpha_{t} \phi-\alpha_{t, h} \phi_{h}\right\|\right)|\psi|_{1} .
\end{aligned}
$$

By the definition of the norm in $H^{-1}(\Omega)$, we conclude that

$$
\begin{aligned}
\left\|\gamma-\gamma_{h}\right\|_{-1}^{2} & \leq\left((2(\mu+\lambda))^{1 / 2}\left\|\phi-\phi_{h}\right\|_{\mathcal{C}}+\left\|\widehat{R e s_{2}}\right\|_{-1}+c_{F} \frac{t^{2}}{12}\left\|\alpha_{t} \phi-\alpha_{t, h} \phi_{h}\right\|\right)^{2} \\
& \leq 6(\mu+\lambda)\left\|\phi-\phi_{h}\right\|_{\mathcal{C}}^{2}+3\left\|\widehat{\operatorname{Res}_{2}}\right\|_{-1}^{2}+3 c_{F}^{2}\left(\frac{t^{2}}{12}\left\|\alpha_{t} \phi-\alpha_{t, h} \phi_{h}\right\|\right)^{2} .
\end{aligned}
$$

\section{Lemma 3.2}

$$
\begin{aligned}
\left\|\phi-\phi_{h}\right\|_{\mathcal{C}}^{2} & +\zeta^{-1} t^{2}\left\|\gamma-\gamma_{h}\right\|^{2}=\widehat{\operatorname{Res}_{1}}\left(\omega-\omega_{h}+z\right)+\widehat{\operatorname{Res}_{2}}\left(\phi-\phi_{h}+\beta\right)-a\left(\phi-\phi_{h}, \beta\right) \\
& +\left(\alpha_{t} \omega-\alpha_{t, h} \omega_{h}, \omega-\omega_{h}+z\right)+\frac{t^{2}}{12}\left(\alpha_{t} \phi-\alpha_{t, h} \phi_{h}, \phi-\phi_{h}+\beta\right),
\end{aligned}
$$

where $z$ and $\beta$ are the functions appearing in the Helmholtz decomposition (6).

Proof: First, (2) and (6) lead to

$$
\begin{aligned}
\left(\gamma-\gamma_{h},\left(\mathbf{R}_{h}-I\right) \phi_{h}\right) & =\left(\gamma-\gamma_{h}, \nabla z-\beta\right) \\
& =(\gamma, \nabla z-\beta)-\left(\gamma_{h}, \nabla z-\beta\right) \\
& =\alpha_{t}(\omega, z)+\alpha_{t} \frac{t^{2}}{12}(\phi, \beta)-a(\phi, \beta)-\left(\gamma_{h}, \nabla z-\beta\right) \\
& =\alpha_{t}(\omega, z)+\alpha_{t} \frac{t^{2}}{12}(\phi, \beta)-a\left(\phi-\phi_{h}, \beta\right)-a\left(\phi_{h}, \beta\right)-\left(\gamma_{h}, \nabla z-\beta\right) .
\end{aligned}
$$


As $\gamma=\zeta t^{-2}(\nabla \omega-\phi)$ and $\gamma_{h}=\zeta t^{-2}\left(\nabla \omega_{h}-\mathbf{R}_{h} \phi_{h}\right)$, we may write $\left\|\phi-\phi_{h}\right\|_{\mathcal{C}}^{2}+\zeta^{-1} t^{2}\left\|\gamma-\gamma_{h}\right\|^{2}=a\left(\phi-\phi_{h}, \phi-\phi_{h}\right)+\left(\gamma-\gamma_{h},\left(\nabla \omega-\nabla \omega_{h}\right)-\left(\phi-\phi_{h}\right)\right)+\left(\gamma-\gamma_{h},\left(\mathbf{R}_{h}-I\right) \phi_{h}\right)$.

By the previous identity and the definition of $\widehat{R e s_{1}}$ and $\widehat{R e s_{2}}$, we obtain

$$
\begin{aligned}
\| \phi & -\phi_{h}\left\|_{\mathcal{C}}^{2}+\zeta^{-1} t^{2}\right\| \gamma-\gamma_{h} \|^{2} \\
= & \alpha_{t}\left(\omega, \omega-\omega_{h}\right)+\alpha_{t} \frac{t^{2}}{12}\left(\phi, \phi-\phi_{h}\right)-a\left(\phi_{h}, \phi-\phi_{h}\right)-\left(\gamma_{h}, \nabla\left(\omega-\omega_{h}\right)\right) \\
& +\left(\gamma_{h}, \phi-\phi_{h}\right)+\alpha_{t}(\omega, z)+\alpha_{t} \frac{t^{2}}{12}(\phi, \beta)-a\left(\phi-\phi_{h}, \beta\right)-a\left(\phi_{h}, \beta\right)-\left(\gamma_{h}, \nabla z-\beta\right) \\
= & \widehat{\operatorname{Res}_{2}}\left(\phi-\phi_{h}+\beta\right)-\frac{t^{2}}{12}\left(\alpha_{t, h} \phi_{h}, \phi-\phi_{h}+\beta\right)-\left(\gamma_{h}, \nabla\left(\omega-\omega_{h}+z\right)\right)-a\left(\phi-\phi_{h}, \beta\right) \\
& +\alpha_{t}\left(\omega, \omega-\omega_{h}+z\right)+\frac{t^{2}}{12}\left(\alpha_{t} \phi, \phi-\phi_{h}+\beta\right) \\
= & \widehat{\operatorname{Res}_{2}}\left(\phi-\phi_{h}+\beta\right)+\widehat{\operatorname{Res}}\left(\omega-\omega_{h}+z\right)-\left(\alpha_{t, h} \omega_{h}, \omega-\omega_{h}+z\right)-a\left(\phi-\phi_{h}, \beta\right) \\
& +\alpha_{t}\left(\omega, \omega-\omega_{h}+z\right)+\frac{t^{2}}{12}\left(\alpha_{t} \phi-\alpha_{t, h} \phi_{h}, \phi-\phi_{h}+\beta\right)+\alpha_{t}\left(\omega, \omega-\omega_{h}+z\right) \\
= & \widehat{\operatorname{Res}_{2}}\left(\phi-\phi_{h}+\beta\right)+\widehat{\operatorname{Res}_{1}}\left(\omega-\omega_{h}+z\right)-a\left(\phi-\phi_{h}, \beta\right) \\
& +\left(\alpha_{t} \omega-\alpha_{t, h} \omega_{h}, \omega-\omega_{h}+z\right)+\frac{t^{2}}{12}\left(\alpha_{t} \phi-\alpha_{t, h} \phi_{h}, \phi-\phi_{h}+\beta\right) .
\end{aligned}
$$

This proves the requested identity.

\section{Lemma 3.3}

$$
\begin{aligned}
& \frac{1}{2}\left\|\phi-\phi_{h}+\beta\right\|_{\mathcal{C}}^{2}+\frac{1}{2}\left\|\phi-\phi_{h}\right\|_{\mathcal{C}}^{2}+\frac{1}{2} \zeta^{-1} t^{2}\left\|\gamma-\gamma_{h}\right\|^{2} \\
& +\frac{1}{2} \sum_{T \in \mathcal{T}_{h}} \frac{\zeta}{t^{2}+h_{T}^{2}}\left\|\nabla\left(\omega-\omega_{h}+z\right)-\left(\phi-\phi_{h}+\beta\right)\right\|_{T}^{2} \\
& \leq\left(\alpha_{t} \omega-\alpha_{t, h} \omega_{h}, \omega-\omega_{h}+z\right)+\frac{t^{2}}{12}\left(\alpha_{t} \phi-\phi_{t, h} \phi_{h}, \phi-\phi_{h}+\beta\right) \\
& \quad \widehat{\operatorname{Res}_{1}}\left(\omega-\omega_{h}+z\right)+\widehat{\operatorname{Res}_{2}}\left(\phi-\phi_{h}+\beta\right)+\frac{1}{2}\|\beta\|_{\mathcal{C}}^{2} .
\end{aligned}
$$

Proof: Because of (6), we first remark that

$$
\gamma-\gamma_{h}=\zeta t^{-2}\left(\nabla \omega-\nabla \omega_{h}-\phi+\phi_{h}+\nabla z-\beta\right),
$$

so that we have for all $T \in \mathcal{T}_{h}$

$$
\left\|\nabla\left(\omega-\omega_{h}+z\right)-\left(\phi-\phi_{h}+\beta\right)\right\|_{T}^{2} \leq \zeta^{-2} t^{4}\left\|\gamma-\gamma_{h}\right\|_{T}^{2} .
$$


This estimate implies that

$$
\begin{aligned}
& \frac{1}{2}\left\|\phi-\phi_{h}+\beta\right\|_{\mathcal{C}}^{2}+\frac{1}{2}\left\|\phi-\phi_{h}\right\|_{\mathcal{C}}^{2}+\frac{1}{2} \zeta^{-1} t^{2}\left\|\gamma-\gamma_{h}\right\|^{2} \\
& \quad+\frac{1}{2} \sum_{T \in \mathcal{T}_{h}} \frac{\zeta}{t^{2}+h_{T}^{2}}\left\|\nabla\left(\omega-\omega_{h}+z\right)-\left(\phi-\phi_{h}+\beta\right)\right\|_{T}^{2} \\
& \leq \frac{1}{2}\left\|\phi-\phi_{h}+\beta\right\|_{\mathcal{C}}^{2}+\frac{1}{2}\left\|\phi-\phi_{h}\right\|_{\mathcal{C}}^{2}+\frac{1}{2} \zeta^{-1} t^{2}\left\|\gamma-\gamma_{h}\right\|^{2}+\frac{1}{2} \zeta^{-1} t^{2} \sum_{T \in \mathcal{T}_{h}}\left\|\gamma-\gamma_{h}\right\|_{T}^{2} \\
& \leq \zeta^{-1} t^{2}\left\|\gamma-\gamma_{h}\right\|^{2}+\frac{1}{2} a\left(\phi-\phi_{h}+\beta, \phi-\phi_{h}+\beta\right)+\frac{1}{2}\left\|\phi-\phi_{h}\right\|_{\mathcal{C}}^{2} \\
& =\zeta^{-1} t^{2}\left\|\gamma-\gamma_{h}\right\|^{2}+\frac{1}{2}\left(\left\|\phi-\phi_{h}\right\|_{\mathcal{C}}^{2}+2 a\left(\phi-\phi_{h}, \beta\right)+\|\beta\|_{\mathcal{C}}^{2}\right)+\frac{1}{2}\left\|\phi-\phi_{h}\right\|_{\mathcal{C}}^{2} \\
& =\left\|\phi-\phi_{h}\right\|_{\mathcal{C}}^{2}+\zeta^{-1} t^{2}\left\|\gamma-\gamma_{h}\right\|^{2}+\frac{1}{2}\|\beta\|_{\mathcal{C}}^{2}+a\left(\phi-\phi_{h}, \beta\right) .
\end{aligned}
$$

The conclusion follows from Lemma 3.2.

\section{Reliability of the estimator}

Theorem 4.1 Let us consider $0<\varepsilon<1 / 2$, as well as two parameters $\nu_{1}>0$ and $\nu_{2}>0$. Moreover, let us define

$$
B(\varepsilon)=\max \left(\frac{3}{\mu}+c_{F}^{2} \frac{\frac{1}{\varepsilon}+\varepsilon-1}{\mu(1-2 \varepsilon)}+6(\mu+\lambda) ; 1+\frac{t^{2}}{\zeta(1-2 \varepsilon)}\right)
$$

Then,

$$
\begin{aligned}
\left(e_{h}^{e v}\right)^{2} \leq & A_{1}\left\|\widehat{\operatorname{Res}_{1}}\right\|_{-1, h}^{2}+A_{2}\left\|\widehat{R e s_{2}}\right\|_{-1}^{2}+A_{3}\left\|\phi-\phi_{h}+\beta\right\|_{\mathcal{C}}^{2}+A_{4}\left\|\phi_{h}-\boldsymbol{R}_{h} \phi_{h}\right\|_{H(r o t, \Omega)}^{2} \\
& -\sum_{T \in \mathcal{T}_{h}} A_{5}^{T}\left\|\nabla\left(\omega-\omega_{h}+z\right)-\left(\phi-\phi_{h}+\beta\right)\right\|_{T}^{2}+A_{6}\left(\frac{t^{2}}{12}\left\|\alpha_{t} \phi-\alpha_{t, h} \phi_{h}\right\|\right)^{2} \\
& +A_{7}\left[\left(\alpha_{t} \omega-\alpha_{t, h} \omega_{h}, \omega-\omega_{h}+z\right)+\frac{t^{2}}{12}\left(\alpha_{t} \phi-\alpha_{t, h} \phi_{h}, \phi-\phi_{h}+\beta\right)\right]
\end{aligned}
$$


with

$$
\mid \begin{aligned}
& A_{1}=\nu_{1} B(\varepsilon)^{2} ; \\
& A_{2}=\nu_{2} B(\varepsilon)^{2}+3 ; \\
& A_{3}=\frac{1}{\mu}\left(\frac{1}{\nu_{1}}+\frac{1}{\nu_{2}}\right)-B(\varepsilon) ; \\
& A_{4}=\max \left(\frac{\frac{2}{\varepsilon}-1}{1-2 \varepsilon} ; 2+2 B(\varepsilon)(\mu+\lambda) c_{R}^{2}\right) ; \\
& A_{5}^{T}=\frac{\zeta B(\varepsilon)}{t^{2}+h_{T}^{2}}-\frac{1}{\nu_{1}\left(t^{2}+h_{T}^{2}\right)}, \forall T \in \mathcal{T}_{h} ; \\
& A_{6}=3 c_{F}^{2} ; \\
& A_{7}=2 B(\varepsilon) .
\end{aligned}
$$

Proof: The proof is similar to the one of [12, Theorem 1] so we only give a sketch of it. Using [12, Lemma 2], we have

$$
\begin{aligned}
\left(e_{h}^{e v}\right)^{2} \leq & \frac{t^{2}}{\zeta(1-2 \varepsilon)} \zeta^{-1} t^{2}\left\|\gamma-\gamma_{h}\right\|^{2}-\frac{1-2 / \varepsilon}{1-2 \varepsilon}\left\|\phi_{h}-\mathbf{R}_{h} \phi_{h}\right\|^{2}-\frac{1-1 / \varepsilon-\varepsilon}{1-2 \varepsilon}\left\|\phi-\phi_{h}\right\|^{2} \\
& +\left|\phi-\phi_{h}\right|_{1}^{2}+\zeta^{-1} t^{2}\left\|\gamma-\gamma_{h}\right\|^{2}+\zeta^{-2} t^{4}\left\|\operatorname{rot}\left(\gamma-\gamma_{h}\right)\right\|^{2}+\left\|\gamma-\gamma_{h}\right\|_{-1}^{2} \\
\leq & \left(1-c_{F}^{2} \frac{1-1 / \varepsilon-\varepsilon}{1-2 \varepsilon}\right)\left|\phi-\phi_{h}\right|_{1}^{2}+\left(1+\frac{t^{2}}{\zeta(1-2 \varepsilon)}\right) \zeta^{-1} t^{2}\left\|\gamma-\gamma_{h}\right\|^{2} \\
& -\frac{1-2 / \varepsilon}{1-2 \varepsilon}\left\|\phi_{h}-\mathbf{R}_{h} \phi_{h}\right\|^{2}+\zeta^{-2} t^{4}\left\|\operatorname{rot}\left(\gamma-\gamma_{h}\right)\right\|^{2}+\left\|\gamma-\gamma_{h}\right\|_{-1}^{2} \\
\leq & \left(\frac{1}{\mu}+c_{F}^{2} \frac{\frac{1}{\varepsilon}+\varepsilon-1}{\mu(1-2 \varepsilon)}\right)\left\|\phi-\phi_{h}\right\|_{\mathcal{C}}^{2}+\left(1+\frac{t^{2}}{\zeta(1-2 \varepsilon)}\right) \zeta^{-1} t^{2}\left\|\gamma-\gamma_{h}\right\|^{2} \\
& +\frac{\frac{2}{\varepsilon}-1}{1-2 \varepsilon}\left\|\phi_{h}-\mathbf{R}_{h} \phi_{h}\right\|^{2}+\zeta^{-2} t^{4}\left\|\operatorname{rot}\left(\gamma-\gamma_{h}\right)\right\|^{2}+\left\|\gamma-\gamma_{h}\right\|_{-1}^{2} .
\end{aligned}
$$

Then, because of Lemma 3.1 as well as

$$
\zeta^{-2} t^{4}\left\|\operatorname{rot}\left(\gamma-\gamma_{h}\right)\right\|^{2} \leq \frac{2}{\mu}\left\|\phi-\phi_{h}\right\|_{\mathcal{C}}^{2}+2\left\|\operatorname{rot}\left(\phi_{h}-\mathbf{R}_{h} \phi_{h}\right)\right\|^{2}
$$

we obtain

$$
\begin{aligned}
\left(e_{h}^{e v}\right)^{2} \leq & \left(\frac{3}{\mu}+c_{F}^{2} \frac{\frac{1}{\varepsilon}+\varepsilon-1}{\mu(1-2 \varepsilon)}+6(\mu+\lambda)\right)\left\|\phi-\phi_{h}\right\|_{\mathcal{C}}^{2}+2\left\|\operatorname{rot}\left(\phi_{h}-\mathbf{R}_{h} \phi_{h}\right)\right\|^{2} \\
& +\left(1+\frac{t^{2}}{\zeta(1-2 \varepsilon)}\right) \zeta^{-1} t^{2}\left\|\gamma-\gamma_{h}\right\|^{2}+\left(\frac{\frac{2}{\varepsilon}-1}{1-2 \varepsilon}\right)\left\|\phi_{h}-\mathbf{R}_{h} \phi_{h}\right\|^{2} \\
& +3\left\|\widehat{R e s_{2}}\right\|_{-1}^{2}+3 c_{F}^{2}\left(\frac{t^{2}}{12}\left\|\alpha_{t} \phi-\alpha_{t, h} \phi_{h}\right\|\right)^{2} .
\end{aligned}
$$


By the definition of $B(\varepsilon)$ as well as Lemma 3.3, we get

$$
\begin{aligned}
\left(e_{h}^{e v}\right)^{2} \leq & B(\varepsilon)\left(2\left(\alpha_{t} \omega-\alpha_{t, h} \omega_{h}, \omega-\omega_{h}+z\right)+2 \frac{t^{2}}{12}\left(\alpha_{t} \phi-\phi_{t, h} \phi_{h}, \phi-\phi_{h}+\beta\right)\right. \\
& +2 \widehat{\operatorname{Res}_{1}}\left(\omega-\omega_{h}+z\right)+2 \widehat{\operatorname{Res}_{2}}\left(\phi-\phi_{h}+\beta\right)+\|\beta\|_{\mathcal{C}}^{2}-\left\|\phi-\phi_{h}+\beta\right\|_{\mathcal{C}}^{2} \\
& \left.-\sum_{T \in \mathcal{T}_{h}} \frac{\zeta}{t^{2}+h_{T}^{2}}\left\|\nabla\left(\omega-\omega_{h}+z\right)-\left(\phi-\phi_{h}+\beta\right)\right\|_{T}^{2}\right)+3\left\|\widehat{\operatorname{Res}_{2}}\right\|_{-1}^{2} \\
& +\left(\frac{\frac{2}{\varepsilon}-1}{1-2 \varepsilon}\right)\left\|\phi_{h}-\mathbf{R}_{h} \phi_{h}\right\|^{2}+2\left\|\operatorname{rot}\left(\phi_{h}-\mathbf{R}_{h} \phi_{h}\right)\right\|^{2} \\
& +3 c_{F}^{2}\left(\frac{t^{2}}{12}\left\|\alpha_{t} \phi-\alpha_{t, h} \phi_{h}\right\|\right)^{2} .
\end{aligned}
$$

We notice that

$$
\begin{aligned}
& \widehat{\operatorname{Res}_{1}}\left(\omega-\omega_{h}+z\right) \leq\left|\left\|\widehat { \operatorname { R e s } _ { 1 } } \left|\left\|_ { - 1 , h } \left|\left\|\left(\psi, \omega-\omega_{h}+z\right) \mid\right\|_{1, h} \forall \psi \in H_{0}^{1}(\Omega)^{2},\right.\right.\right.\right.\right. \\
& \widehat{\operatorname{Res}_{2}}\left(\phi-\phi_{h}+\beta\right) \leq\left\|\widehat{\operatorname{Res}_{2}}\right\|_{-1}\left|\phi-\phi_{h}+\beta\right|_{1} .
\end{aligned}
$$

where, here and below, with a small abuse of notation, we use the extension (by zero) of the linear operator $\widehat{R e s_{1}}$ to the whole of $H_{0}^{1}(\Omega) \times H_{0}^{1}(\Omega)^{2}$ :

$$
\widehat{\operatorname{Res}_{1}}: H_{0}^{1}(\Omega) \times H_{0}^{1}(\Omega)^{2} \longrightarrow \mathbb{R}:(v, \psi) \longmapsto \widehat{\operatorname{Res}_{1}}(v) .
$$

Introducing now the parameters $\nu_{1}>0$ and $\nu_{2}>0$ and using two times Young's inequality lead to

$$
\begin{aligned}
\left(e_{h}^{e v}\right)^{2} \leq & \nu_{1} B(\varepsilon)^{2}\left|\left\|\widehat { R e s _ { 1 } } \left|\left\|_{-1, h}^{2}+\frac{1}{\nu_{1}}\left|\left\|\left(\psi, \omega-\omega_{h}+z\right) \mid\right\|_{1, h}^{2}+\nu_{2} B(\varepsilon)^{2}\left\|\widehat{\operatorname{Res}_{2}}\right\|_{-1}^{2}\right.\right.\right.\right.\right. \\
& +\frac{1}{\nu_{2}}\left|\phi-\phi_{h}+\beta\right|_{1}^{2}-B(\varepsilon)\left\|\phi-\phi_{h}+\beta\right\|_{\mathcal{C}}^{2}+B(\varepsilon)\|\beta\|_{\mathcal{C}}^{2} \\
& +\left(\frac{\frac{2}{\varepsilon}-1}{1-2 \varepsilon}\right)\left\|\phi_{h}-\mathbf{R}_{h} \phi_{h}\right\|^{2}+3\left\|\widehat{R e s_{2}}\right\|_{-1}^{2}+2\left\|\operatorname{rot}\left(\phi_{h}-\mathbf{R}_{h} \phi_{h}\right)\right\|^{2} \\
& -\sum_{T \in \mathcal{T}_{h}}\left(\frac{\zeta B(\varepsilon)}{t^{2}+h_{T}^{2}}\right)\left\|\nabla\left(\omega-\omega_{h}+z\right)-\left(\phi-\phi_{h}+\beta\right)\right\|_{T}^{2} \\
& +2 B(\varepsilon)\left[\left(\alpha_{t} \omega-\alpha_{t, h} \omega_{h}, \omega-\omega_{h}+z\right)+\frac{t^{2}}{12}\left(\alpha_{t} \phi-\alpha_{t, h} \phi_{h}, \phi-\phi_{h}+\beta\right)\right] \\
& +3 c_{F}^{2}\left(\frac{t^{2}}{12}\left\|\alpha_{t} \phi-\alpha_{t, h} \phi_{h}\right\|\right)^{2} .
\end{aligned}
$$

Finally, choosing $\psi=\phi-\phi_{h}+\beta$, we get

$$
\left\|\left(\psi, \omega-\omega_{h}+z\right) \mid\right\|_{1, h}^{2}=\left\|\nabla\left(\phi-\phi_{h}+\beta\right)\right\|^{2}+\sum_{T \in \mathcal{T}_{h}} \frac{1}{t^{2}+h_{T}^{2}}\left\|\nabla\left(\omega-\omega_{h}+z\right)-\left(\phi-\phi_{h}+\beta\right)\right\|_{T}^{2},
$$


and the previous inequality yields (44).

\section{Corollary 4.2 It holds}

$$
\begin{aligned}
& \left(e_{h}^{e v}\right)^{2} \lesssim\left\|\widehat{R e s_{1}} \mid\right\|_{-1, h}^{2}+\left\|\widehat{R e s_{2}}\right\|_{-1}^{2}+\left\|\phi_{h}-\boldsymbol{R}_{h} \phi_{h}\right\|_{H(r o t, \Omega)}^{2} \\
& \left|\left(\alpha_{t} \omega-\alpha_{t, h} \omega_{h}, \omega-\omega_{h}+z\right)+\frac{t^{2}}{12}\left(\alpha_{t} \phi-\alpha_{t, h} \phi_{h}, \phi-\phi_{h}+\beta\right)\right|+t^{4}\left\|\alpha_{t} \phi-\alpha_{t, h} \phi_{h}\right\|^{2} .
\end{aligned}
$$

Proof: Assuming $1-2 \varepsilon>0$, the parameters $\nu_{1}$ and $\nu_{2}$ arising in the values of $A_{3}$ and $A_{5}^{T}$ in (44) are first chosen such that $A_{3} \leq 0$ and $A_{5}^{T} \geq 0$ for all $T \in \mathcal{T}_{h}$. Namely we take $\nu_{1}=\nu_{2}=2 \kappa / B(\varepsilon)$ with

$$
\kappa=\max \left\{\frac{1}{\mu}, \frac{1}{2 \zeta}\right\}
$$

and we obtain

$$
\begin{aligned}
\left(e_{h}^{e v}\right)^{2} \leq & \tilde{A}_{1}\left|\left\|\widehat{R e s_{1}} \mid\right\|_{-1, h}^{2}+\tilde{A}_{2}\left\|\widehat{R e s_{2}}\right\|_{-1}^{2}+\tilde{A}_{4}\left\|\phi_{h}-\mathbf{R}_{h} \phi_{h}\right\|_{H(\text { rot }, \Omega)}^{2}\right. \\
& +\tilde{A}_{6}\left[\left(\alpha_{t} \omega-\alpha_{t, h} \omega_{h}, \omega-\omega_{h}+z\right)+\frac{t^{2}}{12}\left(\alpha_{t} \phi-\alpha_{t, h} \phi_{h}, \phi-\phi_{h}+\beta\right)\right] \\
& +\tilde{A}_{7}\left(\frac{t^{2}}{12}\left\|\alpha_{t} \phi-\alpha_{t, h} \phi_{h}\right\|\right)^{2},
\end{aligned}
$$

with

$$
\mid \begin{aligned}
& \tilde{A}_{1}=2 \kappa B(\varepsilon) ; \\
& \tilde{A}_{2}=2 \kappa B(\varepsilon)+3 ; \\
& \tilde{A}_{4}=\max \left(\frac{\frac{2}{\varepsilon}-1}{1-2 \varepsilon} ; 2+2 B(\varepsilon)(\mu+\lambda) c_{R}^{2}\right) ; \\
& \tilde{A}_{6}=2 B(\varepsilon) ; \\
& \tilde{A}_{7}=3 c_{F}^{2} .
\end{aligned}
$$

We conclude by taking any $\varepsilon \in\left(0, \frac{1}{2}\right)$.

\section{Lemma 4.3}

$$
\begin{aligned}
& \left|\left(\alpha_{t} \omega-\alpha_{t, h} \omega_{h}, \omega-\omega_{h}+z\right)+\frac{t^{2}}{12}\left(\alpha_{t} \phi-\alpha_{t, h} \phi_{h}, \phi-\phi_{h}+\beta\right)\right| \\
& \leq\left(\frac{c_{F}^{2} C}{1+c_{F}^{2}}\right)^{1 / 2}\left(\left\|\alpha_{t} \omega-\alpha_{t, h} \omega_{h}\right\|+\frac{t^{2}}{12}\left\|\alpha_{t} \phi-\alpha_{t, h} \phi_{h}\right\|\right)\left\|\phi_{h}-\boldsymbol{R}_{h} \phi_{h}\right\|_{H(\text { rot }, \Omega)} \\
& +\frac{\alpha_{t}+\alpha_{t, h}}{2}\left(\left\|\omega-\omega_{h}\right\|^{2}+\frac{t^{2}}{12}\left\|\phi-\phi_{h}\right\|^{2}\right) .
\end{aligned}
$$


Proof: Clearly we have

$$
\begin{aligned}
& \left(\alpha_{t} \omega-\alpha_{t, h} \omega_{h}, \omega-\omega_{h}+z\right)+\frac{t^{2}}{12}\left(\alpha_{t} \phi-\alpha_{t, h} \phi_{h}, \phi-\phi_{h}+\beta\right) \\
& =\left(\alpha_{t} \omega-\alpha_{t, h} \omega_{h}, \omega-\omega_{h}\right)+\frac{t^{2}}{12}\left(\alpha_{t} \phi-\alpha_{t, h} \phi_{h}, \phi-\phi_{h}\right) \\
& +\left(\alpha_{t} \omega-\alpha_{t, h} \omega_{h}, z\right)+\frac{t^{2}}{12}\left(\alpha_{t} \phi-\alpha_{t, h} \phi_{h}, \beta\right) .
\end{aligned}
$$

We can notice that for all $v \in H_{0}^{1}(\Omega)$ or $v \in H_{0}^{1}(\Omega)^{2}$, we have

$$
\|v\| \leq\left(\frac{c_{F}^{2}}{1+c_{F}^{2}}\right)^{\frac{1}{2}}\|v\|_{1} .
$$

Using Cauchy-Schwarz's inequality and (7), we have :

$$
\begin{aligned}
& \left|\left(\alpha_{t} \omega-\alpha_{t, h} \omega_{h}, z\right)+\frac{t^{2}}{12}\left(\alpha_{t} \phi-\alpha_{t, h} \phi_{h}, \beta\right)\right| \\
& \leq\left\|\alpha_{t} \omega-\alpha_{t, h} \omega_{h}\right\|\|z\|+\frac{t^{2}}{12}\left\|\alpha_{t} \phi-\alpha_{t, h} \phi_{h}\right\|\|\beta\| \\
& \leq\left\|\alpha_{t} \omega-\alpha_{t, h} \omega_{h}\right\|\left(\frac{c_{F}^{2}}{1+c_{F}^{2}}\right)^{1 / 2}\|z\|_{1}+\frac{t^{2}}{12}\left\|\alpha_{t} \phi-\alpha_{t, h} \phi_{h}\right\|\left(\frac{c_{F}^{2}}{1+c_{F}^{2}}\right)^{1 / 2}\|\beta\|_{1} \\
& \leq\left(\frac{c_{F}^{2} C}{1+c_{F}^{2}}\right)^{1 / 2}\left[\left\|\alpha_{t} \omega-\alpha_{t, h} \omega_{h}\right\|^{2}+\left(\frac{t^{2}}{12}\left\|\alpha_{t} \phi-\alpha_{t, h} \phi_{h}\right\|\right)^{2}\right]^{1 / 2}\left\|\phi_{h}-\mathbf{R}_{h} \phi_{h}\right\|_{H(\text { rot }, \Omega)} .
\end{aligned}
$$

For the other term in the right-hand side of (46), we have by the normalization of the eigenvectors :

$$
\begin{gathered}
\left(\alpha_{t} \omega-\alpha_{t, h} \omega_{h}, \omega-\omega_{h}\right)+\frac{t^{2}}{12}\left(\alpha_{t} \phi-\alpha_{t, h} \phi_{h}, \phi-\phi_{h}\right) \\
=\alpha_{t}\|\omega\|^{2}-\left(\alpha_{t}+\alpha_{t, h}\right)\left(\omega, \omega_{h}\right)+\alpha_{t, h}\left\|\omega_{h}\right\|^{2} \\
\quad+\frac{t^{2}}{12}\left(\alpha_{t}\|\phi\|^{2}-\left(\alpha_{t}+\alpha_{t, h}\right)\left(\phi, \phi_{h}\right)+\alpha_{t, h}\left\|\phi_{h}\right\|^{2}\right) \\
=\left(\alpha_{t}+\alpha_{t, h}\right)\left(1-\left(\omega, \omega_{h}\right)-\frac{t^{2}}{12}\left(\phi, \phi_{h}\right)\right) .
\end{gathered}
$$

But we also have :

$$
\begin{aligned}
\left\|\omega-\omega_{h}\right\|^{2}+\frac{t^{2}}{12}\left\|\phi-\phi_{h}\right\|^{2} & =\|\omega\|^{2}-2\left(\omega, \omega_{h}\right)+\left\|\omega_{h}\right\|^{2}+\frac{t^{2}}{12}\left(\|\phi\|^{2}-2\left(\phi, \phi_{h}\right)+\left\|\phi_{h}\right\|^{2}\right) \\
& =2-2\left(\omega, \omega_{h}\right)-2 \frac{t^{2}}{12}\left(\phi, \phi_{h}\right) .
\end{aligned}
$$


Hence :

$$
\left(\alpha_{t} \omega-\alpha_{t, h} \omega_{h}, \omega-\omega_{h}\right)+\frac{t^{2}}{12}\left(\alpha_{t} \phi-\alpha_{t, h} \phi_{h}, \phi-\phi_{h}\right)=\frac{\alpha_{t}+\alpha_{t, h}}{2}\left(\left\|\omega-\omega_{h}\right\|^{2}+\frac{t^{2}}{12}\left\|\phi-\phi_{h}\right\|^{2}\right) .
$$

Using (47) and (49) into (46), Lemma 4.3 holds.

Now, it remains to bound each of the two residuals.

Lemma 4.4 With the notations (9) and (12), we have

$$
\left|\left\|\widehat{R e s_{1}} \mid\right\|_{-1, h}^{2} \lesssim \sum_{T \in \mathcal{T}_{h}} h_{T}^{2}\left(t^{2}+h_{T}^{2}\right)\left\|\alpha_{t, h} \omega_{h}+\operatorname{div} \gamma_{h}\right\|_{T}^{2}+\sum_{E \in \mathcal{E}_{h}} h_{E}\left(t^{2}+h_{E}^{2}\right)\left\|\left[\gamma_{h}\right]_{E} \cdot n_{E}\right\|_{E}^{2} .\right.
$$

where $\left[\gamma_{h}\right]_{E}$ is the jump of $\gamma_{h}$ across $E$ defined by :

$$
\left[\gamma_{h}\right]_{E}=\left.\gamma_{h}\right|_{T^{+}}-\left.\gamma_{h}\right|_{T^{-}} \text {with } E=\overline{T^{+}} \cap \overline{T^{-}} .
$$

Proof: Let $v \in H_{0}^{1}(\Omega)$. Using standard Green formula into each element of the triangulation, we get :

$$
\begin{aligned}
\widehat{\operatorname{Res}_{1}}(v) & =\left(\alpha_{t, h} \omega_{h}, v\right)-\left(\gamma_{h}, \nabla v\right) \\
& =\sum_{T \in \mathcal{T}_{h}}\left[\int_{T}\left(\alpha_{t, h} \omega_{h}+d i v \gamma_{h}\right) v-\sum_{E \in \partial T} \int_{E} \gamma_{h} \cdot n_{E} v\right] \\
& =\sum_{T \in \mathcal{T}_{h}} \int_{T}\left(\alpha_{t, h} \omega_{h}+\operatorname{div} \gamma_{h}\right) v-\sum_{E \in \mathcal{E}_{h}} \int_{E}\left[\gamma_{h}\right]_{E} \cdot n_{E} v
\end{aligned}
$$

Let $v^{I} \in S_{0}^{1}\left(\mathcal{T}_{h}\right)=\left\{v \in H_{0}^{1}(\Omega): \forall T \in \mathcal{T}_{h},\left.v\right|_{T} \in \mathbb{P}_{1}(T)\right\}$ (cf. estimate (3.6) of [10]) be such that, for all $T \in \mathcal{T}_{h}$ :

$$
\begin{cases}\left\|v-v^{I}\right\|_{T} \lesssim h_{T}\left\{\|\nabla v-\psi\|_{\omega_{T}}+h_{T}\|\nabla \psi\|_{\omega_{T}}\right\} & \forall \psi \in H_{0}^{1}(\Omega)^{2} \\ \left\|v-v^{I}\right\|_{E} \lesssim h_{E}^{1 / 2}\left\{\|\nabla v-\psi\|_{\omega_{E}}+h_{E}\|\nabla \psi\|_{\omega_{E}}\right\} & \forall \psi \in H_{0}^{1}(\Omega)^{2}\end{cases}
$$

We can notice that $\widehat{\operatorname{Res}_{1}}\left(v^{I}\right)=0$, and consequently :

$$
\begin{aligned}
\widehat{\operatorname{Res}_{1}}(v) & =\widehat{R e s_{1}}\left(v-v^{I}\right) \\
& =\sum_{T \in \mathcal{T}_{h}} \int_{T}\left(\alpha_{t, h} \omega_{h}+\operatorname{div} \gamma_{h}\right)\left(v-v^{I}\right)-\sum_{E \in \mathcal{E}_{h}} \int_{E}\left[\gamma_{h}\right]_{E} \cdot n_{E}\left(v-v^{I}\right) \\
& \leq \sum_{T \in \mathcal{T}_{h}}\left\|\alpha_{t, h} \omega_{h}+\operatorname{div} \gamma_{h}\right\|_{T}\left\|v-v^{I}\right\|_{T}+\sum_{E \in \mathcal{E}_{h}}\left\|\left[\gamma_{h}\right]_{E} \cdot n_{E}\right\|_{E}\left\|v-v^{I}\right\|_{E} \\
& \lesssim \sum_{T \in \mathcal{T}_{h}} h_{T} \sqrt{t^{2}+h_{T}^{2}}\left\|\alpha_{t, h} \omega_{h}+\operatorname{div} \gamma_{h}\right\|_{T} \frac{1}{\sqrt{t^{2}+h_{T}^{2}}}\left\{\|\nabla v-\psi\|_{\omega_{T}}+h_{T}\|\nabla \psi\|_{\omega_{T}}\right\}
\end{aligned}
$$




$$
\begin{aligned}
&+\sum_{E \in \mathcal{E}_{h}} h_{E}^{1 / 2} \sqrt{t^{2}+h_{E}^{2}}\left\|\left[\gamma_{h}\right]_{E} \cdot n_{E}\right\|_{E} \frac{1}{\sqrt{t^{2}+h_{E}^{2}}}\left\{\|\nabla v-\psi\|_{\omega_{E}}+h_{T}\|\nabla \psi\|_{\omega_{E}}\right\} \\
& \lesssim\left(\sum_{T \in \mathcal{T}_{h}} h_{T}^{2}\left(t^{2}+h_{T}^{2}\right)\left\|\alpha_{t, h} \omega_{h}+\operatorname{div} \gamma_{h}\right\|_{T}^{2}\right)^{1 / 2}\left|\|(\psi, v) \mid\|_{1, h}\right. \\
&+\left(\sum_{E \in \mathcal{E}_{h}} h_{E}\left(t^{2}+h_{E}^{2}\right)\left\|\left[\gamma_{h}\right]_{E} \cdot n_{E}\right\|_{E}^{2}\right)^{1 / 2}\left|\|(\psi, v) \mid\|_{1, h},\right.
\end{aligned}
$$

so that (50) holds.

Lemma 4.5 With the notation (10), we have

$$
\begin{array}{r}
\left\|\widehat{R e s_{2}}\right\|_{-1}^{2} \lesssim \sum_{T \in \mathcal{T}_{h}} h_{T}^{2}\left\|\operatorname{div} \mathcal{C} \varepsilon\left(\phi_{h}\right)+\gamma_{h}+\frac{t^{2}}{12} \alpha_{t, h} \phi_{h}\right\|_{T}^{2} \\
+\sum_{E \in \mathcal{E}_{h}} h_{E}\left\|\left[\mathcal{C} \varepsilon\left(\phi_{h}\right)\right]_{E} n_{E}\right\|_{E}^{2}+\mu_{h}^{2}\left(\gamma_{h}\right)
\end{array}
$$

where $\mu_{h}\left(\gamma_{h}\right)=\sup _{\eta_{h} \in S_{0}^{1}\left(\mathcal{T}_{h}\right)^{2} \backslash\{0\}} \frac{\left|\left(\gamma_{h},\left(\boldsymbol{I}-\boldsymbol{R}_{h}\right) \eta_{h}\right)\right|}{\left|\eta_{h}\right|_{1}}$.

Proof: Let $\psi \in \Theta$. Using standard Green formula into each element of the triangulation, we get :

$$
\begin{aligned}
\widehat{\operatorname{Res}_{2}}(\psi) & =-a\left(\phi_{h}, \psi\right)+\left(\gamma_{h}, \psi\right)+\frac{t^{2}}{12}\left(\alpha_{t, h} \phi_{h}, \psi\right) \\
& =\sum_{T \in \mathcal{T}_{h}}\left[\int_{T}\left(\operatorname{div} \mathcal{C} \varepsilon\left(\phi_{h}\right)+\gamma_{h}+\frac{t^{2}}{12} \alpha_{t, h} \phi_{h}\right) \cdot \psi-\sum_{E \in \partial T} \int_{E} \mathcal{C} \varepsilon\left(\phi_{h}\right) n_{E} \cdot \psi\right] \\
& =\sum_{T \in \mathcal{T}_{h}} \int_{T}\left(\operatorname{div} \mathcal{C} \varepsilon\left(\phi_{h}\right)+\gamma_{h}+\frac{t^{2}}{12} \alpha_{t, h} \phi_{h}\right) \cdot \psi-\sum_{E \in \mathcal{E}_{h}} \int_{E}\left[\mathcal{C} \varepsilon\left(\phi_{h}\right)\right]_{E} n_{E} \cdot \psi
\end{aligned}
$$

Let $\psi^{I} \in S_{0}^{1}\left(\mathcal{T}_{h}\right)^{2} \subset \Theta_{h}$ (cf. [1, Theorem 1.7]) be such that $\left|\psi^{I}\right|_{1} \lesssim|\psi|_{1}$ and for all $T \in \mathcal{T}_{h}$

$$
\left\{\begin{aligned}
\left\|\psi-\psi^{I}\right\|_{T} \lesssim h_{T}\|\nabla \psi\|_{\omega_{T}} \\
\left\|\psi-\psi^{I}\right\|_{E} \lesssim h_{E}^{1 / 2}\|\nabla \psi\|_{\omega_{E}}
\end{aligned}\right.
$$

We can notice that $\widehat{\operatorname{Res}_{2}}\left(\psi^{I}\right)=\left(\gamma_{h},\left(\mathbf{I}-\mathbf{R}_{h}\right) \psi^{I}\right)$, which implies :

$$
\widehat{\operatorname{Res}_{2}}(\psi)=\widehat{\operatorname{Res}_{2}}\left(\psi-\psi^{I}\right)+\left(\gamma_{h},\left(\mathbf{I}-\mathbf{R}_{h}\right) \psi^{I}\right)
$$




$$
\begin{aligned}
& =\sum_{T \in \mathcal{T}_{h}} \int_{T}\left(\operatorname{div} \mathcal{C} \varepsilon\left(\phi_{h}\right)+\gamma_{h}+\frac{t^{2}}{12} \alpha_{t, h} \phi_{h}\right) \cdot\left(\psi-\psi^{I}\right) \\
& \quad-\sum_{E \in \mathcal{E}_{h}} \int_{E}\left[\mathcal{C} \varepsilon\left(\phi_{h}\right)\right]_{E} n_{E} \cdot\left(\psi-\psi^{I}\right)+\left(\gamma_{h},\left(\mathbf{I}-\mathbf{R}_{h}\right) \psi^{I}\right) \\
& \leq \sum_{T \in \mathcal{T}_{h}}\left\|\operatorname{div} \mathcal{C} \varepsilon\left(\phi_{h}\right)+\gamma_{h}+\frac{t^{2}}{12} \alpha_{t, h} \phi_{h}\right\|_{T}\left\|\psi-\psi^{I}\right\|_{T} \\
& \quad+\sum_{E \in \mathcal{E}_{h}}\left\|\left[\mathcal{C} \varepsilon\left(\phi_{h}\right)\right]_{E} n_{E}\right\|_{E}\left\|\psi-\psi^{I}\right\|_{E}+\frac{\left|\left(\gamma_{h},\left(\mathbf{I}-\mathbf{R}_{h}\right) \psi^{I}\right)\right|}{\left|\psi^{I}\right|_{1}}\left|\psi^{I}\right|_{1} \\
& \lesssim \sum_{T \in \mathcal{T}_{h}} h_{T}\left\|\operatorname{div} \mathcal{C} \varepsilon\left(\phi_{h}\right)+\gamma_{h}+\frac{t^{2}}{12} \alpha_{t, h} \phi_{h}\right\|_{T}\|\nabla \psi\|_{\omega_{T}} \\
& +\sum_{E \in \mathcal{E}_{h}} h_{E}^{1 / 2}\left\|\left[\mathcal{C} \varepsilon\left(\phi_{h}\right)\right]_{E} n_{E}\right\|_{E}\|\nabla \psi\|_{\omega_{E}}+\mu_{h}\left(\gamma_{h}\right)\left|\psi^{I}\right|_{1} .
\end{aligned}
$$

Then, using the estimate $\left|\psi^{I}\right|_{1} \lesssim|\psi|_{1}$ and the discrete Cauchy-Schwarz inequality, we get

$$
\begin{aligned}
\widehat{\operatorname{Res}_{2}}(\psi) \lesssim( & \left.\sum_{T \in \mathcal{T}_{h}} h_{T}^{2}\left\|\operatorname{div} \mathcal{C} \varepsilon\left(\phi_{h}\right)+\gamma_{h}+\frac{t^{2}}{12} \alpha_{t, h} \phi_{h}\right\|_{T}^{2}\right)^{1 / 2}|\psi|_{1} \\
& +\left(\sum_{E \in \mathcal{E}_{h}} h_{E}\left\|\left[\mathcal{C} \varepsilon\left(\phi_{h}\right)\right]_{E} n_{E}\right\|_{E}^{2}\right)^{1 / 2}|\psi|_{1}+\mu_{h}\left(\gamma_{h}\right)|\psi|_{1} .
\end{aligned}
$$

The definition of the norm of $H^{-1}(\Omega)$ leads to (51).

Theorem 4.6 We have

$$
\begin{aligned}
& \left(e_{h}^{e v}\right)^{2} \lesssim \sum_{T \in \mathcal{T}_{h}} h_{T}^{2}\left(t^{2}+h_{T}^{2}\right)\left\|\alpha_{t, h} \omega_{h}+\operatorname{div} \gamma_{h}\right\|_{T}^{2}+\sum_{E \in \mathcal{E}_{h}} h_{E}\left(t^{2}+h_{E}^{2}\right)\left\|\left[\gamma_{h}\right]_{E} \cdot n_{E}\right\|_{E}^{2} \\
& \quad+\sum_{T \in \mathcal{T}_{h}} h_{T}^{2}\left\|\operatorname{div} \mathcal{C} \varepsilon\left(\phi_{h}\right)+\gamma_{h}+\frac{t^{2}}{12} \alpha_{t, h} \phi_{h}\right\|_{T}^{2}+\sum_{E \in \mathcal{E}_{h}} h_{E}\left\|\left[\mathcal{C} \varepsilon\left(\phi_{h}\right)\right]_{E} n_{E}\right\|_{E}^{2} \\
& \quad+\left\|\phi_{h}-\boldsymbol{R}_{h} \phi_{h}\right\|_{H(r o t, \Omega)}^{2}+\mu_{h}\left(\gamma_{h}\right)^{2}+\left(\frac{t^{2}}{12}\left\|\alpha_{t} \phi-\alpha_{t, h} \phi_{h}\right\|\right)^{2} \\
& \quad+\left\|\alpha_{t} \omega-\alpha_{t, h} \omega_{h}\right\|^{2}+\frac{\alpha_{t}+\alpha_{t, h}}{2}\left(\left\|\omega-\omega_{h}\right\|^{2}+\frac{t^{2}}{12}\left\|\phi-\phi_{h}\right\|^{2}\right) .
\end{aligned}
$$

Proof: The theorem is a direct consequence of Corollary 4.2, Lemma 4.3, Lemma 4.4 and Lemma 4.5.

Remark 4.7 From a practical point of view, the estimate (53) is not useful since the $L^{2}(\Omega)$ norm of the error is still present in the right-hand-side (see theorem 3.1 of [19] for a similar phenomenom for the Laplace equation). However, the terms containing the exact solution in the right hand side of (53) are neglectible if the eigenvalue is simple (cf. Theorem 2.4). This is the subject of the following corollary. 
Corollary 4.8 (Reliability of the estimator) Assume that $\alpha_{t}$ is a simple eigenvalue, then we have:

$$
\begin{aligned}
\left(e_{h}^{e v}\right)^{2} \lesssim \sum_{T \in \mathcal{T}_{h}} h_{T}^{2}\left(t^{2}+h_{T}^{2}\right)\left\|\alpha_{t, h} \omega_{h}+\operatorname{div} \gamma_{h}\right\|_{T}^{2}+\sum_{E \in \mathcal{E}(\Omega)} h_{E}\left(t^{2}+h_{E}^{2}\right)\left\|\left[\gamma_{h}\right]_{E} \cdot n_{E}\right\|_{E}^{2} \\
\quad+\sum_{T \in \mathcal{T}_{h}} h_{T}^{2}\left\|\operatorname{div} \mathcal{C} \varepsilon\left(\phi_{h}\right)+\gamma_{h}+\frac{t^{2}}{12} \alpha_{t, h} \phi_{h}\right\|_{T}^{2}+\sum_{E \in \mathcal{E}(\Omega)} h_{E}\left\|\left[\mathcal{C} \varepsilon\left(\phi_{h}\right)\right]_{E} n_{E}\right\|_{E}^{2} \\
+\left\|\phi_{h}-\boldsymbol{R}_{h} \phi_{h}\right\|_{H(\text { rot }, \Omega)}^{2}+\mu_{h}\left(\gamma_{h}\right)^{2}+\text { h.o.t. }
\end{aligned}
$$

where h.o.t. corresponds to higher order terms.

Proof: Using Cauchy-Schwarz's inequality, we get :

$$
\begin{aligned}
& \left(\frac{t^{2}}{12}\left\|\alpha_{t} \phi-\alpha_{t, h} \phi_{h}\right\|\right)^{2}+\left\|\alpha_{t} \omega-\alpha_{t, h} \omega_{h}\right\|^{2} \\
& =\left(\alpha_{t} \omega-\alpha_{t, h} \omega_{h}, \alpha_{t}\left(\omega-\omega_{h}\right)+\left(\alpha_{t}-\alpha_{t, h}\right) \omega_{h}\right) \\
& \quad+\left(\frac{t^{2}}{12}\right)^{2}\left(\alpha_{t} \phi-\alpha_{t, h} \phi_{h}, \alpha_{t}\left(\phi-\phi_{h}\right)+\left(\alpha_{t}-\alpha_{t, h}\right) \phi_{h}\right) \\
& \leq \alpha_{t}\left(\alpha_{t} \omega-\alpha_{t, h} \omega_{h}, \omega-\omega_{h}\right)+\alpha_{t}\left(\frac{t^{2}}{12}\right)^{2}\left(\alpha_{t} \phi-\alpha_{t, h} \phi_{h}, \phi-\phi_{h}\right) \\
& \quad+\left|\alpha_{t}-\alpha_{t, h}\right|\left\{\left\|\alpha_{t} \omega-\alpha_{t, h} \omega_{h}\right\|\left\|\omega_{h}\right\|+\left(\frac{t^{2}}{12}\right)^{2}\left\|\alpha_{t} \phi-\alpha_{t, h} \phi_{h}\right\|\left\|\phi_{h}\right\|\right\} .
\end{aligned}
$$

But, by (49), we have :

$$
\begin{aligned}
& \left(\alpha_{t} \omega-\alpha_{t, h} \omega_{h}, \omega-\omega_{h}\right)+\left(\frac{t^{2}}{12}\right)^{2}\left(\alpha_{t} \phi-\alpha_{t, h} \phi_{h}, \phi-\phi_{h}\right) \\
& \quad \leq \max \left\{1 ; \frac{t^{2}}{12}\right\}\left[\begin{array}{l}
\left.\left(\alpha_{t} \omega-\alpha_{t, h} \omega_{h}, \omega-\omega_{h}\right)+\frac{t^{2}}{12}\left(\alpha_{t} \phi-\alpha_{t, h} \phi_{h}, \phi-\phi_{h}\right)\right] \\
\leq \max \left\{1 ; \frac{t^{2}}{12}\right\} \frac{\alpha_{t}+\alpha_{t, h}}{2}\left[\left\|\omega-\omega_{h}\right\|^{2}+\frac{t^{2}}{12}\left\|\phi-\phi_{h}\right\|^{2}\right] .
\end{array}\right.
\end{aligned}
$$

By a discrete Cauchy-Schwarz inequality and the normalization of the discrete solutions given by (4), we get:

$$
\begin{aligned}
& \left\|\alpha_{t} \omega-\alpha_{t, h} \omega_{h}\right\|\left\|\omega_{h}\right\|+\left(\frac{t^{2}}{12}\right)^{2}\left\|\alpha_{t} \phi-\alpha_{t, h} \phi_{h}\right\|\left\|\phi_{h}\right\| \\
& \leq\left(\left\|\alpha_{t} \omega-\alpha_{t, h} \omega_{h}\right\|^{2}+\left(\frac{t^{2}}{12}\right)^{2}\left\|\alpha_{t} \phi-\alpha_{t, h} \phi_{h}\right\|^{2}\right)^{1 / 2}\left(\left\|\omega_{h}\right\|^{2}+\left(\frac{t^{2}}{12}\right)^{2}\left\|\phi_{h}\right\|^{2}\right)^{1 / 2} \\
& \leq\left(\left\|\alpha_{t} \omega-\alpha_{t, h} \omega_{h}\right\|^{2}+\left(\frac{t^{2}}{12}\right)^{2}\left\|\alpha_{t} \phi-\alpha_{t, h} \phi_{h}\right\|^{2}\right)^{1 / 2} \max \left\{1 ; \frac{t^{2}}{12}\right\}^{1 / 2} .
\end{aligned}
$$


Therefore, using Young's inequality with a parameter $\delta>0$ :

$$
\begin{aligned}
& \left(\frac{t^{2}}{12}\left\|\alpha_{t} \phi-\alpha_{t, h} \phi_{h}\right\|\right)^{2}+\left\|\alpha_{t} \omega-\alpha_{t, h} \omega_{h}\right\|^{2} \\
& \leq \alpha_{t} \max \left\{1 ; \frac{t^{2}}{12}\right\} \frac{\alpha_{t}+\alpha_{t, h}}{2}\left[\left\|\omega-\omega_{h}\right\|^{2}+\frac{t^{2}}{12}\left\|\phi-\phi_{h}\right\|^{2}\right] \\
& \quad+\left|\alpha_{t}-\alpha_{t, h}\right| \max \left\{1 ; \frac{t^{2}}{12}\right\}^{1 / 2}\left(\left\|\alpha_{t} \omega-\alpha_{t, h} \omega_{h}\right\|^{2}+\left(\frac{t^{2}}{12}\right)^{2}\left\|\alpha_{t} \phi-\alpha_{t, h} \phi_{h}\right\|^{2}\right)^{1 / 2} \\
& \leq \alpha_{t} \max \left\{1 ; \frac{t^{2}}{12}\right\} \frac{\alpha_{t}+\alpha_{t, h}}{2}\left[\left\|\omega-\omega_{h}\right\|^{2}+\frac{t^{2}}{12}\left\|\phi-\phi_{h}\right\|^{2}\right] \\
& \quad+\frac{\delta}{2}\left|\alpha_{t}-\alpha_{t, h}\right|^{2} \max \left\{1 ; \frac{t^{2}}{12}\right\}+\frac{1}{2 \delta}\left(\left\|\alpha_{t} \omega-\alpha_{t, h} \omega_{h}\right\|^{2}+\left(\frac{t^{2}}{12}\right)^{2}\left\|\alpha_{t} \phi-\alpha_{t, h} \phi_{h}\right\|^{2}\right) .
\end{aligned}
$$

Choosing $\delta=1$, we get :

$$
\begin{aligned}
& \left(\frac{t^{2}}{12}\left\|\alpha_{t} \phi-\alpha_{t, h} \phi_{h}\right\|\right)^{2}+\left\|\alpha_{t} \omega-\alpha_{t, h} \omega_{h}\right\|^{2} \\
& \leq \alpha_{t}\left(\alpha_{t}+\alpha_{t, h}\right) \max \left\{1 ; \frac{t^{2}}{12}\right\}\left[\left\|\omega-\omega_{h}\right\|^{2}+\frac{t^{2}}{12}\left\|\phi-\phi_{h}\right\|^{2}\right]+\left|\alpha_{t}-\alpha_{t, h}\right|^{2} \max \left\{1 ; \frac{t^{2}}{12}\right\} .
\end{aligned}
$$

As $\left\|\omega-\omega_{h}\right\|^{2},\left\|\phi-\phi_{h}\right\|^{2}$ and $\left|\alpha_{t}-\alpha_{t, h}\right|^{2}$ are superconvergent (cf. Theorem 2.4), then $\left(\frac{t^{2}}{12}\left\|\alpha_{t} \phi-\alpha_{t, h} \phi_{h}\right\|\right)^{2}+\left\|\alpha_{t} \omega-\alpha_{t, h} \omega_{h}\right\|^{2}+\frac{\alpha_{t}+\alpha_{t, h}}{2}\left(\left\|\omega-\omega_{h}\right\|^{2}+\frac{t^{2}}{12}\left\|\phi-\phi_{h}\right\|^{2}\right)$ are superconvergent.

\section{A posteriori estimate for the eigenvalue error}

Let $\eta_{e v}$ be the estimator presented in Corollary 4.8 :

$$
\begin{aligned}
\eta_{e v}^{2} & =\sum_{T \in \mathcal{T}_{h}} h_{T}^{2}\left(t^{2}+h_{T}^{2}\right)\left\|\alpha_{t, h} \omega_{h}+\operatorname{div} \gamma_{h}\right\|_{T}^{2}+\sum_{E \in \mathcal{E}_{h}} h_{E}\left(t^{2}+h_{E}^{2}\right)\left\|\left[\gamma_{h}\right]_{E} \cdot n_{E}\right\|_{E}^{2} \\
& +\sum_{T \in \mathcal{T}_{h}} h_{T}^{2}\left\|\operatorname{div} \mathcal{C} \varepsilon\left(\phi_{h}\right)+\gamma_{h}+\frac{t^{2}}{12} \alpha_{t, h} \phi_{h}\right\|_{T}^{2}+\sum_{E \in \mathcal{E}_{h}} h_{E}\left\|\left[\mathcal{C} \varepsilon\left(\phi_{h}\right)\right]_{E} n_{E}\right\|_{E}^{2} \\
& +\left\|\phi_{h}-\mathbf{R}_{h} \phi_{h}\right\|_{H(\text { rot }, \Omega)}^{2}+\mu_{h}\left(\gamma_{h}\right)^{2} .
\end{aligned}
$$

Theorem 5.1 We have:

$$
\left|\alpha_{t}-\alpha_{t, h}\right| \lesssim \eta_{e v}^{2}+\zeta t^{-2}\left\|\phi_{h}-\boldsymbol{R}_{h} \phi_{h}\right\|^{2}+\left(\gamma_{h}, \phi_{h}-\boldsymbol{R}_{h} \phi_{h}\right)+T_{e x}^{2},
$$

where $T_{e x}^{2}=\left(\frac{t^{2}}{12}\left\|\alpha_{t} \phi-\alpha_{t, h} \phi_{h}\right\|\right)^{2}+\left\|\alpha_{t} \omega-\alpha_{t, h} \omega_{h}\right\|^{2}+\frac{\alpha_{t}+\alpha_{t, h}}{2}\left(\left\|\omega-\omega_{h}\right\|^{2}+\frac{t^{2}}{12}\left\|\phi-\phi_{h}\right\|^{2}\right)$ is the term containing the exact solution. 
Proof: We recall that $\left(\omega, \phi, \alpha_{t}\right)$ (resp. $\left.\left(\omega_{h}, \phi_{h}, \alpha_{t, h}\right)\right)$ is the solution of problem (2) (resp. (4)). Then we have :

$$
\begin{aligned}
\| \phi & -\phi_{h}\left\|_{\mathcal{C}}^{2}+\zeta^{-1} t^{2}\right\| \gamma-\gamma_{h} \|^{2} \\
& =\|\phi\|_{\mathcal{C}}^{2}+\left\|\phi_{h}\right\|_{\mathcal{C}}^{2}-2 a\left(\phi, \phi_{h}\right)+\zeta^{-1} t^{2}\left(\|\gamma\|^{2}+\left\|\gamma_{h}\right\|^{2}-2\left(\gamma, \gamma_{h}\right)\right) \\
& =\|\phi\|_{\mathcal{C}}^{2}+\zeta^{-1} t^{2}\|\gamma\|^{2}+\left\|\phi_{h}\right\|_{\mathcal{C}}^{2}+\zeta^{-1} t^{2}\left\|\gamma_{h}\right\|^{2}-2\left(a\left(\phi, \phi_{h}\right)+\zeta^{-1} t^{2}\left(\gamma, \gamma_{h}\right)\right) \\
& =\alpha_{t}+\alpha_{t, h}-2\left(a\left(\phi, \phi_{h}\right)+\zeta^{-1} t^{2}\left(\gamma, \gamma_{h}\right)\right)
\end{aligned}
$$

as well as

$$
\begin{aligned}
a\left(\phi, \phi_{h}\right)+\zeta^{-1} t^{2}\left(\gamma, \gamma_{h}\right) & =a\left(\phi, \phi_{h}\right)+\left(\gamma, \nabla \omega_{h}-\mathbf{R}_{h} \phi_{h}\right) \\
& =a\left(\phi, \phi_{h}\right)+\left(\gamma, \nabla \omega_{h}-\phi_{h}\right)+\left(\gamma, \phi_{h}-\mathbf{R}_{h} \phi_{h}\right) \\
& =\alpha_{t}\left[\left(\omega, \omega_{h}\right)+\frac{t^{2}}{12}\left(\phi, \phi_{h}\right)\right]+\left(\gamma, \phi_{h}-\mathbf{R}_{h} \phi_{h}\right)
\end{aligned}
$$

Then, from the relations (55) and (56), we have :

$$
\left\|\phi-\phi_{h}\right\|_{\mathcal{C}}^{2}+\zeta^{-1} t^{2}\left\|\gamma-\gamma_{h}\right\|^{2}=\alpha_{t}+\alpha_{t, h}-2\left(\alpha_{t}\left[\left(\omega, \omega_{h}\right)+\frac{t^{2}}{12}\left(\phi, \phi_{h}\right)\right]+\left(\gamma, \phi_{h}-\mathbf{R}_{h} \phi_{h}\right)\right)
$$

so that from (48) :

$$
\begin{aligned}
& \left\|\phi-\phi_{h}\right\|_{\mathcal{C}}^{2}+\zeta^{-1} t^{2}\left\|\gamma-\gamma_{h}\right\|^{2} \\
& \quad=\alpha_{t}+\alpha_{t, h}+\alpha_{t}\left[\left\|\omega-\omega_{h}\right\|^{2}+\frac{t^{2}}{12}\left\|\phi-\phi_{h}\right\|^{2}-2\right]-2\left(\gamma, \phi_{h}-\mathbf{R}_{h} \phi_{h}\right) \\
& =\alpha_{t, h}-\alpha_{t}+\alpha_{t}\left[\left\|\omega-\omega_{h}\right\|^{2}+\frac{t^{2}}{12}\left\|\phi-\phi_{h}\right\|^{2}\right]-2\left(\gamma, \phi_{h}-\mathbf{R}_{h} \phi_{h}\right),
\end{aligned}
$$

In other words, noticing that $\alpha_{t}>0$, we have : 


$$
\begin{aligned}
& \alpha_{t, h}-\alpha_{t}=\left\|\phi-\phi_{h}\right\|_{\mathcal{C}}^{2}+\zeta^{-1} t^{2}\left\|\gamma-\gamma_{h}\right\|^{2}-\alpha_{t}\left[\left\|\omega-\omega_{h}\right\|^{2}+\frac{t^{2}}{12}\left\|\phi-\phi_{h}\right\|^{2}\right] \\
& +2\left(\gamma, \phi_{h}-\mathbf{R}_{h} \phi_{h}\right) \\
& =\left\|\phi-\phi_{h}\right\|_{\mathcal{C}}^{2}+\zeta^{-1} t^{2}\left\|\gamma-\gamma_{h}\right\|^{2}-\alpha_{t}\left[\left\|\omega-\omega_{h}\right\|^{2}+\frac{t^{2}}{12}\left\|\phi-\phi_{h}\right\|^{2}\right] \\
& +2\left(\gamma-\gamma_{h}, \phi_{h}-\mathbf{R}_{h} \phi_{h}\right)+2\left(\gamma_{h}, \phi_{h}-\mathbf{R}_{h} \phi_{h}\right) \\
& \leq\left\|\phi-\phi_{h}\right\|_{\mathcal{C}}^{2}+\zeta^{-1} t^{2}\left\|\gamma-\gamma_{h}\right\|^{2}-\alpha_{t}\left[\left\|\omega-\omega_{h}\right\|^{2}+\frac{t^{2}}{12}\left\|\phi-\phi_{h}\right\|^{2}\right] \\
& +2\left\|\gamma-\gamma_{h}\right\|\left\|\phi_{h}-\mathbf{R}_{h} \phi_{h}\right\|+2\left(\gamma_{h}, \phi_{h}-\mathbf{R}_{h} \phi_{h}\right) \\
& \leq\left\|\phi-\phi_{h}\right\|_{\mathcal{C}}^{2}+\zeta^{-1} t^{2}\left\|\gamma-\gamma_{h}\right\|^{2}-\alpha_{t}\left[\left\|\omega-\omega_{h}\right\|^{2}+\frac{t^{2}}{12}\left\|\phi-\phi_{h}\right\|^{2}\right] \\
& +\zeta^{-1} t^{2}\left\|\gamma-\gamma_{h}\right\|^{2}+\zeta t^{-2}\left\|\phi_{h}-\mathbf{R}_{h} \phi_{h}\right\|^{2}+2\left(\gamma_{h}, \phi_{h}-\mathbf{R}_{h} \phi_{h}\right) \\
& \leq 2\left[\left\|\phi-\phi_{h}\right\|_{\mathcal{C}}^{2}+\zeta^{-1} t^{2}\left\|\gamma-\gamma_{h}\right\|^{2}\right]-\alpha_{t}\left[\left\|\omega-\omega_{h}\right\|^{2}+\frac{t^{2}}{12}\left\|\phi-\phi_{h}\right\|^{2}\right] \\
& +\zeta t^{-2}\left\|\phi_{h}-\mathbf{R}_{h} \phi_{h}\right\|^{2}+2\left(\gamma_{h}, \phi_{h}-\mathbf{R}_{h} \phi_{h}\right) \\
& \leq 2\left(e_{h}^{e v}\right)^{2}+\zeta t^{-2}\left\|\phi_{h}-\mathbf{R}_{h} \phi_{h}\right\|^{2}+2\left(\gamma_{h}, \phi_{h}-\mathbf{R}_{h} \phi_{h}\right) \text {. }
\end{aligned}
$$

Using Theorem 4.6, we obtain :

$$
\alpha_{t, h}-\alpha_{t} \leq C_{1}\left(\eta_{e v}^{2}+T_{e x}^{2}\right)+\zeta t^{-2}\left\|\phi_{h}-\mathbf{R}_{h} \phi_{h}\right\|^{2}+2\left(\gamma_{h}, \phi_{h}-\mathbf{R}_{h} \phi_{h}\right),
$$

for some $C_{1}>0$ (independent of $t$ and $h$ ).

In order to obtain an evaluation of the error eigenvalues, we must now evaluate $\alpha_{t}-\alpha_{t, h}$ to finally control the quantity $\left|\alpha_{t}-\alpha_{t, h}\right|$. All we have to do is to repeat the previous arguments replacing (56) by the identity

$$
a\left(\phi, \phi_{h}\right)+\zeta^{-1} t^{2}\left(\gamma, \gamma_{h}\right)=-\widehat{\operatorname{Res}_{1}}(\omega)-\widehat{\operatorname{Res}_{2}}(\phi)+\alpha_{t, h}\left[\left(\omega_{h}, \omega\right)+\frac{t^{2}}{12}\left(\phi_{h}, \phi\right)\right],
$$

that directly follows from the definition of $\widehat{R e s_{1}}$ and $\widehat{R e s_{2}}$. Furthermore by (2) and (4), we see that

$$
\alpha_{t}=a(\phi, \phi)+\zeta^{-1} t^{2}\|\gamma\|^{2}, \quad \alpha_{t, h}=a\left(\phi_{h}, \phi_{h}\right)+\zeta^{-1} t^{2}\left\|\gamma_{h}\right\|^{2} .
$$

These two identities and the normalization in (2) and (4) lead to

$$
\begin{aligned}
\alpha_{t}-\alpha_{t, h}=\| \phi & -\phi_{h}\left\|_{\mathcal{C}}^{2}+\zeta^{-1} t^{2}\right\| \gamma-\gamma_{h} \|^{2}-\alpha_{t, h}\left[\left\|\omega-\omega_{h}\right\|^{2}+\frac{t^{2}}{12}\left\|\phi-\phi_{h}\right\|^{2}\right] \\
& -2 \widehat{\operatorname{Res}_{1}}(\omega)-2 \widehat{\operatorname{Res}_{2}}(\phi) .
\end{aligned}
$$

Then, using the fact that $\widehat{\operatorname{Res}_{1}}\left(\omega_{h}\right)+\widehat{\operatorname{Res}_{2}}\left(\phi_{h}\right)=\left(\gamma_{h}, \phi_{h}-\mathbf{R}_{h} \phi_{h}\right)$ and inserting the functions $\beta$ and $z$ from the Helmholtz decomposition (6) we get

$$
\alpha_{t}-\alpha_{t, h}=\left\|\phi-\phi_{h}\right\|_{\mathcal{C}}^{2}+\zeta^{-1} t^{2}\left\|\gamma-\gamma_{h}\right\|^{2}-\alpha_{t, h}\left[\left\|\omega-\omega_{h}\right\|^{2}+\frac{t^{2}}{12}\left\|\phi-\phi_{h}\right\|^{2}\right]
$$




$$
\begin{aligned}
& -2\left(\widehat{\operatorname{Res}_{1}}\left(\omega-\omega_{h}+z\right)+\left(\gamma_{h}, \nabla z\right)-\left(\alpha_{t, h} \omega_{h}, z\right)\right) \\
& -2\left(\widehat{\operatorname{Res}_{2}}\left(\phi-\phi_{h}+\beta\right)+\left(\gamma_{h}, \phi_{h}-\mathbf{R}_{h} \phi_{h}\right)+a\left(\phi_{h}, \beta\right)-\left(\gamma_{h}, \beta\right)-\frac{t^{2}}{12}\left(\alpha_{t, h} \phi_{h}, \beta\right)\right) \\
=\| \phi & -\phi_{h}\left\|_{\mathcal{C}}^{2}+\zeta^{-1} t^{2}\right\| \gamma-\gamma_{h} \|^{2}-\alpha_{t, h}\left[\left\|\omega-\omega_{h}\right\|^{2}+\frac{t^{2}}{12}\left\|\phi-\phi_{h}\right\|^{2}\right] \\
& -2\left(\widehat{\operatorname{Res}_{1}}\left(\omega-\omega_{h}+z\right)+\widehat{\operatorname{Res}_{2}}\left(\phi-\phi_{h}+\beta\right)+\left(\gamma_{h}, \phi_{h}-\mathbf{R}_{h} \phi_{h}\right)\right. \\
& \left.+a\left(\phi_{h}-\phi, \beta\right)+\left(\gamma-\gamma_{h}, \phi_{h}-\mathbf{R}_{h} \phi_{h}\right)+\left(\alpha_{t} \omega-\alpha_{t, h} \omega_{h}, z\right)+\frac{t^{2}}{12}\left(\alpha_{t} \phi-\alpha_{t, h} \phi_{h}, \beta\right)\right),
\end{aligned}
$$

the last identity following from (2). By Cauchy-Schwarz's inequality, we get

$$
\begin{aligned}
\alpha_{t}-\alpha_{t, h} \leq \| \phi & -\phi_{h}\left\|_{\mathcal{C}}^{2}+\zeta^{-1} t^{2}\right\| \gamma-\gamma_{h} \|^{2}-\alpha_{t, h}\left[\left\|\omega-\omega_{h}\right\|^{2}+\frac{t^{2}}{12}\left\|\phi-\phi_{h}\right\|^{2}\right] \\
& +2\left\{\left|\widehat{R e s_{1}}\left(\omega-\omega_{h}+z\right)\right|+\left|\widehat{\operatorname{Res}_{2}}\left(\phi-\phi_{h}+\beta\right)\right|+\left|\left(\gamma_{h}, \phi_{h}-\mathbf{R}_{h} \phi_{h}\right)\right|\right. \\
& +\left\|\phi_{h}-\phi\right\|_{\mathcal{C}}\|\beta\|_{\mathcal{C}}+\zeta^{-1 / 2} t\left\|\gamma-\gamma_{h}\right\| \zeta^{1 / 2} t^{-1}\left\|\phi_{h}-\mathbf{R}_{h} \phi_{h}\right\| \\
+ & \left.\left(\left\|\alpha_{t} \omega-\alpha_{t, h} \omega_{h}\right\|^{2}+\left(\frac{t^{2}}{12}\right)^{2}\left\|\alpha_{t} \phi-\alpha_{t, h} \phi_{h}\right\|^{2}\right)^{1 / 2}\left(\|z\|^{2}+\|\beta\|^{2}\right)^{1 / 2}\right\} .
\end{aligned}
$$

Noticing that $\alpha_{t, h}>0$ and using the reliability of the estimator presented in section 4 we obtain :

$$
\alpha_{t}-\alpha_{t, h} \leq C_{2}\left(\eta_{e v}^{2}+T_{e x}^{2}\right)+\zeta t^{-2}\left\|\phi_{h}-\mathbf{R}_{h} \phi_{h}\right\|^{2}+2\left(\gamma_{h}, \phi_{h}-\mathbf{R}_{h} \phi_{h}\right),
$$

for some $C_{2}>0$ (independent of $t$ and $h$ ). Hence (54) is a direct consequence of the estimates (57) and (58).

Similarly to Corollary 4.8, we have :

Corollary 5.2 Assume that $\alpha_{t}$ is a simple eigenvalue, then we have:

$$
\left|\alpha_{t}-\alpha_{t, h}\right| \lesssim \eta_{e v}^{2}+\zeta t^{-2}\left\|\phi_{h}-\boldsymbol{R}_{h} \phi_{h}\right\|^{2}+\left(\gamma_{h}, \phi_{h}-\boldsymbol{R}_{h} \phi_{h}\right)+\text { h.o.t. }
$$

Remark 5.3 The term $\left(\gamma_{h}, \phi_{h}-\boldsymbol{R}_{h} \phi_{h}\right)$ can be evaluated numerically. However, it can be bounded by $\left\|\gamma_{h}\right\|\left\|\phi_{h}-\boldsymbol{R}_{h} \phi_{h}\right\|$. We further can numerically remark that the term $\| \phi_{h}-$ $\boldsymbol{R}_{h} \phi_{h} \|$ converges faster than the estimator: hence if $\alpha_{t}$ is a simple eigenvalue, we can claim that the term $\left|\alpha_{t}-\alpha_{t, h}\right|$ is superconvergent (since it is bounded by the square of $\eta_{\text {ev }}$ up to higher order terms) and the relation (iii) given in Theorem 2.4 is recovered. 


\section{Efficiency of the estimator}

In order to prove the efficiency of the estimator, each part of it (except the terms involving the exact solutions) has now to be bounded by the error $e_{h}^{e v}$ up to a multiplicative constant.

\section{Lemma 6.1}

$$
\begin{aligned}
\left\|\left(\boldsymbol{R}_{h}-I\right) \phi_{h}\right\|_{H(r o t, \Omega)}^{2} \lesssim \zeta^{-2} t^{4} \| \gamma & -\gamma_{h} \|_{\Omega}^{2}+\left|\omega-\omega_{h}\right|_{1}^{2} \\
& +\left|\phi-\phi_{h}\right|_{1}^{2}+\zeta^{-2} t^{4}\left\|\operatorname{rot}\left(\gamma-\gamma_{h}\right)\right\|^{2} .
\end{aligned}
$$

Proof: Since

$$
\left(\mathbf{R}_{h}-I\right) \phi_{h}=\zeta^{-1} t^{2}\left(\gamma-\gamma_{h}\right)-\nabla\left(\omega-\omega_{h}\right)+\left(\phi-\phi_{h}\right)
$$

we have

$$
\left.\| \mathbf{R}_{h}-I\right) \phi_{h}\left\|\leq \zeta^{-1} t^{2}\right\| \gamma-\gamma_{h}\left\|+\left|\omega-\omega_{h}\right|_{1}+\right\| \phi-\phi_{h} \|,
$$

and with the Poincaré-Friedrichs inequality, we get

$$
\left\|\left(\mathbf{R}_{h}-I\right) \phi_{h}\right\|^{2} \lesssim \zeta^{-2} t^{4}\left\|\gamma-\gamma_{h}\right\|^{2}+\left|\omega-\omega_{h}\right|_{1}^{2}+\left|\phi-\phi_{h}\right|_{1}^{2}
$$

Moreover, we have

$$
\left\|\operatorname{rot}\left(\phi_{h}-\mathbf{R}_{h} \phi_{h}\right)\right\|^{2} \lesssim \zeta^{-2} t^{4}\left\|\operatorname{rot}\left(\gamma-\gamma_{h}\right)\right\|^{2}+\left|\phi-\phi_{h}\right|_{1}^{2}
$$

so that lemma 6.1 holds.

Lemma 6.2 We have

$$
\begin{array}{r}
\sum_{T \in \mathcal{T}_{h}} h_{T}^{2}\left(t^{2}+h_{T}^{2}\right)\left\|\alpha_{t, h} \omega_{h}+\operatorname{div} \gamma_{h}\right\|_{T}^{2} \lesssim \sum_{T \in \mathcal{T}_{h}} h_{T}^{2}\left(t^{2}+h_{T}^{2}\right)\left\|\alpha_{t, h} \omega_{h}-\alpha_{t} \omega\right\|_{T}^{2} \\
+t^{2}\left\|\gamma-\gamma_{h}\right\|^{2}+\left\|\gamma-\gamma_{h}\right\|_{-1}^{2} .
\end{array}
$$

and

$$
\begin{array}{r}
\sum_{E \in \mathcal{E}(\Omega)} h_{E}\left(h_{E}^{2}+t^{2}\right)\left\|\left[\gamma_{h}\right]_{E} \cdot n_{E}\right\|_{E}^{2} \lesssim \sum_{T \in \mathcal{T}_{h}} h_{T}^{2}\left(t^{2}+h_{T}^{2}\right)\left\|\alpha_{t} \omega-\alpha_{t, h} \omega_{h}\right\|_{T}^{2} \\
+t^{2}\left\|\gamma-\gamma_{h}\right\|^{2}+\left\|\gamma-\gamma_{h}\right\|_{-1}^{2} .
\end{array}
$$

Proof: Let $v_{T}=b_{T}^{2}\left(\alpha_{t, h} \omega_{h}+\operatorname{div} \gamma_{h}\right)$ for all $T \in \mathcal{T}_{h}, b_{T}$ being the classical element bubble function. So, we get by the elementwise inverse estimates :

$$
\begin{aligned}
\left\|\alpha_{t, h} \omega_{h}+\operatorname{div} \gamma_{h}\right\|_{T}^{2} & \lesssim\left(\alpha_{t, h} \omega_{h}+\operatorname{div} \gamma_{h}, v_{T}\right)_{T} \\
& =\left(\alpha_{t, h} \omega_{h}, v_{T}\right)_{T}-\left(\gamma_{h}, \nabla v_{T}\right)_{T} \\
& =\left(\alpha_{t, h} \omega_{h}-\alpha_{t} \omega, v_{T}\right)_{T}+\left(\gamma-\gamma_{h}, \nabla v_{T}\right)_{T} .
\end{aligned}
$$


By summation and Cauchy-Schwarz inequalities, we get

$$
\begin{aligned}
\sum_{T \in \mathcal{T}_{h}} & h_{T}^{2}\left(t^{2}+h_{T}^{2}\right)\left\|\alpha_{t, h} \omega_{h}+\operatorname{div} \gamma_{h}\right\|_{T}^{2} \\
& =\sum_{T \in \mathcal{T}_{h}} h_{T}^{2}\left(t^{2}+h_{T}^{2}\right)\left(\alpha_{t, h} \omega_{h}-\alpha_{t} \omega, v_{T}\right)_{T}+\sum_{T \in \mathcal{T}_{h}} h_{T}^{2}\left(t^{2}+h_{T}^{2}\right)\left(\gamma-\gamma_{h}, \nabla v_{T}\right)_{T} \\
& \leq \sum_{T \in \mathcal{T}_{h}} h_{T} \sqrt{t^{2}+h_{T}^{2}}\left\|\alpha_{t, h} \omega_{h}-\alpha_{t} \omega\right\|_{T} h_{T} \sqrt{t^{2}+h_{T}^{2}}\left\|v_{T}\right\|_{T}+\sum_{T \in \mathcal{T}_{h}} t\left\|\gamma-\gamma_{h}\right\|_{T} t h_{T}^{2}\left\|\nabla v_{T}\right\|_{T} \\
& +\left\|\gamma-\gamma_{h}\right\|_{-1}\left|\sum_{T \in \mathcal{T}_{h}} h_{T}^{4} \nabla v_{T}\right|_{1} .
\end{aligned}
$$

Using the elementwise inverse estimates :

$$
\begin{aligned}
\left\|\nabla v_{T}\right\|_{T} & \lesssim h_{T}^{-1}\left\|\alpha_{t, h} \omega_{h}+\operatorname{div} \gamma_{h}\right\|_{\omega_{T}} \\
\left|\nabla v_{T}\right|_{1, T} & \lesssim h_{T}^{-2}\left\|\alpha_{t, h} \omega_{h}+\operatorname{div} \gamma_{h}\right\|_{\omega_{T}}
\end{aligned}
$$

and notifying that

$$
\left|\sum_{T \in \mathcal{T}_{h}} h_{T}^{4} \nabla v_{T}\right|_{1}^{2}=\sum_{T \in \mathcal{T}_{h}} h_{T}^{8}\left|\nabla v_{T}\right|_{1, T}^{2} \lesssim \sum_{T \in \mathcal{T}_{h}} h_{T}^{4}\left\|\alpha_{t, h} \omega_{h}+d i v \gamma_{h}\right\|_{\omega_{T}}^{2},
$$

we obtain, using the regularity of the mesh and discrete Cauchy-Schwarz inequalities in (62)

$$
\begin{aligned}
\sum_{T \in \mathcal{T}_{h}} & h_{T}^{2}\left(t^{2}+h_{T}^{2}\right)\left\|\alpha_{t, h} \omega_{h}+\operatorname{div} \gamma_{h}\right\|_{T}^{2} \\
& \lesssim\left(\sum_{T \in \mathcal{T}_{h}} h_{T}^{2}\left(t^{2}+h_{T}^{2}\right)\left\|\alpha_{t, h} \omega_{h}-\alpha_{t} \omega\right\|_{T}^{2}\right)^{1 / 2}\left(\sum_{T \in \mathcal{T}_{h}} h_{T}^{2}\left(t^{2}+h_{T}^{2}\right)\left\|\alpha_{t, h} \omega_{h}+\operatorname{div} \gamma_{h}\right\|_{T}^{2}\right)^{1 / 2} \\
& +t\left\|\gamma-\gamma_{h}\right\|\left(\sum_{T \in \mathcal{T}_{h}} h_{T}^{2} t^{2}\left\|\alpha_{t, h} \omega_{h}-\alpha_{t} \omega\right\|_{T}^{2}\right)^{1 / 2}+\left\|\gamma-\gamma_{h}\right\|_{-1}\left(\sum_{T \in \mathcal{T}_{h}} h_{T}^{4}\left\|\alpha_{t, h} \omega_{h}-\alpha_{t} \omega\right\|_{T}^{2}\right)^{1 / 2} .
\end{aligned}
$$

Using Young's inequality in this last estimate, we get (59).

For all interior edge $E=\overline{T_{+}} \cap \overline{T_{-}}$, we define the classical edge bubble function $b_{E} \in H_{0}^{1}\left(\omega_{E}\right)$ such as

$$
\mid \begin{array}{ll}
\operatorname{supp} b_{E}=\overline{\omega_{E}}, & 0 \leq b_{E} \leq 1=\max _{x \in E} b_{E}, \\
\int_{\omega_{E}} b_{E} d x \approx h_{E}^{2}, & \int_{E} b_{E} d s \approx h_{E}, \\
\left\|\nabla b_{E}\right\|_{L^{2}\left(T_{ \pm}\right)} \lesssim h_{E}^{-1}\left\|b_{E}\right\|_{L^{2}\left(T_{ \pm}\right)}, & \left|\nabla b_{E}\right|_{H^{1}\left(\omega_{E}\right)} \lesssim h_{E}^{-2}\left\|b_{E}\right\|_{L^{2}\left(\omega_{E}\right)} .
\end{array}
$$


For all $E \in \mathcal{E}_{h}$, we define $w_{E}=b_{E}^{2}\left(\left[\gamma_{h}\right]_{E} \cdot n_{E}\right) \in H_{0}^{2}\left(\omega_{E}\right)^{2}$ with $\left\|w_{E}\right\|_{\omega_{E}}^{2} \lesssim h_{E}\left\|\left[\gamma_{h}\right]_{E} \cdot n_{E}\right\|_{E}^{2}$. So, we obtain :

$$
\begin{aligned}
\left\|\left[\gamma_{h}\right]_{E} \cdot n_{E}\right\|_{E}^{2} & \lesssim\left(\left[\gamma_{h}\right]_{E} \cdot n_{E}, w_{E}\right)_{E} \\
& =\left(\operatorname{div} \gamma_{h}, w_{E}\right)_{\omega_{E}}+\left(\gamma_{h}, \nabla w_{E}\right)_{\omega_{E}} \\
& =\left(\alpha_{t, h} \omega_{h}+\operatorname{div} \gamma_{h}, w_{E}\right)_{\omega_{E}}+\left(\gamma_{h}, \nabla w_{E}\right)_{\omega_{E}}-\left(\alpha_{t, h} \omega_{h}, w_{E}\right)_{\omega_{E}} \\
& =\left(\alpha_{t, h} \omega_{h}+\operatorname{div} \gamma_{h}, w_{E}\right)_{\omega_{E}}-\left(\gamma-\gamma_{h}, \nabla w_{E}\right)_{\omega_{E}}+\left(\alpha_{t} \omega-\alpha_{t, h} \omega_{h}, w_{E}\right)_{\omega_{E}} .
\end{aligned}
$$

Using a discrete Cauchy-Schwarz inequality and the regularity of the mesh, we have by summation

$$
\begin{aligned}
& \sum_{E \in \mathcal{E}_{h}} h_{E}\left(h_{E}^{2}+t^{2}\right)\left\|\left[\gamma_{h}\right]_{E} \cdot n_{E}\right\|_{E}^{2} \\
& \lesssim \sum_{E \in \mathcal{E}_{h}}\left\{\left(\alpha_{t, h} \omega_{h}+d i v \gamma_{h}, h_{E}\left(h_{E}^{2}+t^{2}\right) w_{E}\right)_{\omega_{E}}+\left(\alpha_{t} \omega-\alpha_{t, h} \omega_{h}, h_{E}\left(h_{E}^{2}+t^{2}\right) w_{E}\right)_{\omega_{E}}\right\} \\
& \quad-\left(\gamma-\gamma_{h}, \sum_{E \in \mathcal{E}_{h}} h_{E}\left(h_{E}^{2}+t^{2}\right) \nabla w_{E}\right){ }_{\omega_{E}}{ }^{1 / 2}\left(\sum_{E \in \mathcal{E}_{h}}\left(h_{E}^{2}+t^{2}\right)\left\|w_{E}\right\|_{\omega_{E}}^{2}\right)^{1 / 2} \\
& \lesssim\left(\sum_{T \in \mathcal{T}_{h}} h_{T}^{2}\left(t^{2}+h_{T}^{2}\right)\left\|\alpha_{t, h} \omega_{h}+d i v \gamma_{h}\right\|_{T}^{2}\right)^{1 / 2} \\
& \quad+\left(\sum_{T \in \mathcal{T}_{h}} h_{T}^{2}\left(t^{2}+h_{T}^{2}\right)\left\|\alpha_{t} \omega-\alpha_{t, h} \omega_{h}\right\|_{T}^{2}\right)^{1 / 2}\left(\sum_{E \in \mathcal{E}_{h}}\left(h_{E}^{2}+t^{2}\right)\left\|w_{E}\right\|_{\omega_{E}}^{2}\right)^{1 / 2}\|\|_{i} t h_{E} \nabla w_{E} \| . \\
& \left.\quad+\left\|\gamma-\gamma_{h}\right\|_{-1} h_{E \in \mathcal{E}(\Omega)} h_{E}^{3} \nabla w_{E}\right)\|t\| \gamma-\gamma_{h} \|
\end{aligned}
$$

By the following inverse estimate :

$$
\left\|\nabla w_{E}\right\|_{\omega_{E}}+h_{E}\left|\nabla w_{E}\right|_{1, \omega_{E}} \lesssim h_{E}^{-1 / 2}\left\|\left[\gamma_{h}\right]_{E} \cdot n_{E}\right\|_{E},
$$

we get :

$$
\begin{aligned}
\left\|\nabla\left(\sum_{E \in \mathcal{E}_{h}} h_{E}^{3} \nabla w_{E}\right)\right\|^{2} & \lesssim \sum_{E \in \mathcal{E}_{h}} h_{E}^{6}\left|\nabla w_{E}\right|_{1}^{2} \lesssim \sum_{E \in \mathcal{E}_{h}} h_{E}^{3}\left\|\left[\gamma_{h}\right]_{E} \cdot n_{E}\right\|_{E}^{2} \\
& \leq \sum_{E \in \mathcal{E}_{h}} h_{E}\left(t^{2}+h_{E}^{2}\right)\left\|\left[\gamma_{h}\right]_{E} \cdot n_{E}\right\|_{E}^{2} .
\end{aligned}
$$

The same kind of argument give :

$$
\begin{aligned}
\left\|\sum_{E \in \mathcal{E}_{h}} t h_{E} \nabla w_{E}\right\|^{2} & \lesssim \sum_{E \in \mathcal{E}_{h}} t^{2} h_{E}^{2}\left\|\nabla w_{E}\right\|_{\omega_{E}}^{2} \lesssim \sum_{E \in \mathcal{E}_{h}} t^{2} h_{E}\left\|\left[\gamma_{h}\right]_{E} \cdot n_{E}\right\|_{E}^{2} \\
& \leq \sum_{E \in \mathcal{E}_{h}} h_{E}\left(t^{2}+h_{E}^{2}\right)\left\|\left[\gamma_{h}\right]_{E} \cdot n_{E}\right\|_{E}^{2} .
\end{aligned}
$$


By (65), (66) and (67), we obtain :

$$
\begin{gathered}
\sum_{E \in \mathcal{E}_{h}} h_{E}\left(h_{E}^{2}+t^{2}\right)\left\|\left[\gamma_{h}\right]_{E} \cdot n_{E}\right\|_{E}^{2} \lesssim \sum_{T \in \mathcal{T}_{h}} h_{T}^{2}\left(t^{2}+h_{T}^{2}\right)\left\|\alpha_{t, h} \omega_{h}+\operatorname{div} \gamma_{h}\right\|_{T}^{2}+\left\|\gamma-\gamma_{h}\right\|_{-1}^{2} \\
+t^{2}\left\|\gamma-\gamma_{h}\right\|^{2}+\sum_{T \in \mathcal{T}_{h}} h_{T}^{2}\left(t^{2}+h_{T}^{2}\right)\left\|\alpha_{t} \omega-\alpha_{t, h} \omega_{h}\right\|_{T}^{2}
\end{gathered}
$$

Using (59) in (68), we get (60).

Lemma 6.3 We have

$$
\begin{aligned}
\sum_{T \in \mathcal{T}_{h}} h_{T}^{2}\left\|\operatorname{div} \mathcal{C} \varepsilon\left(\phi_{h}\right)+\gamma_{h}+\frac{t^{2}}{12} \alpha_{t, h} \phi_{h}\right\|_{T}^{2} \lesssim & \left|\phi-\phi_{h}\right|_{1}^{2}+\left\|\gamma-\gamma_{h}\right\|_{-1}^{2} \\
& +\frac{t^{2}}{12} \sum_{T \in \mathcal{T}_{h}} h_{T}^{2}\left\|\alpha_{t} \phi-\alpha_{t, h} \phi_{h}\right\|_{T}^{2},
\end{aligned}
$$

and

$$
\sum_{E \in \mathcal{E}_{h}} h_{E}\left\|\left[\mathcal{C} \varepsilon\left(\phi_{h}\right)\right]_{E} n_{E}\right\|_{E}^{2} \lesssim\left|\phi-\phi_{h}\right|_{1}^{2}+\left\|\gamma-\gamma_{h}\right\|_{-1}^{2}+\frac{t^{2}}{12} \sum_{T \in \mathcal{T}_{h}} h_{T}^{2}\left\|\alpha_{t} \phi-\alpha_{t, h} \phi_{h}\right\|_{T}^{2} .
$$

Proof: We just need to use the same arguments as the proof of lemma 6.2.

\section{Lemma 6.4}

$$
\mu_{h}\left(\gamma_{h}\right)^{2} \lesssim\left\|\gamma-\gamma_{h}\right\|_{-1}^{2}+\left|\phi-\phi_{h}\right|_{1}^{2}+\left(\frac{t^{2}}{12}\left\|\alpha_{t, h} \phi_{h}-\alpha_{t} \phi\right\|\right)^{2}
$$

Proof: We recall the definition of $\mu_{h}\left(\gamma_{h}\right)$ :

$$
\mu_{h}\left(\gamma_{h}\right)=\sup _{\eta_{h} \in S_{0}^{1}\left(\mathcal{T}_{h}\right)^{2} \backslash\{0\}} \frac{\left|\left(\gamma_{h},\left(\mathbf{I}-\mathbf{R}_{h}\right) \eta_{h}\right)\right|}{\left|\eta_{h}\right|_{1}} .
$$

Let $\eta_{h} \in S_{0}^{1}\left(\mathcal{T}_{h}\right)^{2} \backslash\{0\}$. Although,

$$
\begin{aligned}
\left(\gamma_{h},\left(\mathbf{I}-\mathbf{R}_{h}\right) \eta_{h}\right) & =\left(\gamma_{h}, \eta_{h}\right)-\left(\gamma_{h}, \mathbf{R}_{h} \eta_{h}\right) \\
& =\left(\gamma_{h}, \eta_{h}\right)+\alpha_{t, h} \frac{t^{2}}{12}\left(\phi_{h}, \eta_{h}\right)-a\left(\phi_{h}, \eta_{h}\right) \\
& =\left(\gamma_{h}-\gamma, \eta_{h}\right)+\frac{t^{2}}{12}\left(\alpha_{t, h} \phi_{h}-\alpha_{t} \phi, \eta_{h}\right)+a\left(\phi-\phi_{h}, \eta_{h}\right) \\
& \leq\left\|\gamma-\gamma_{h}\right\|_{-1}\left|\eta_{h}\right|_{1}+\left\|\phi-\phi_{h}\right\|_{\mathcal{C}}\left\|\eta_{h}\right\|_{\mathcal{C}} \\
& +\frac{t^{2}}{12}\left\|\alpha_{t, h} \phi_{h}-\alpha_{t} \phi\right\|\left\|\eta_{h}\right\| .
\end{aligned}
$$

The Korn and Friedrichs inequalities lead to (71). 
Theorem 6.5 We have

$$
\begin{aligned}
& \sum_{T \in \mathcal{T}_{h}} h_{T}^{2}\left(t^{2}+h_{T}^{2}\right)\left\|\alpha_{t, h} \omega_{h}+\operatorname{div} \gamma_{h}\right\|_{T}^{2}+\sum_{E \in \mathcal{E}_{h}} h_{E}\left(t^{2}+h_{E}^{2}\right)\left\|\left[\gamma_{h}\right]_{E} \cdot n_{E}\right\|_{E}^{2}+\left\|\phi_{h}-\boldsymbol{R}_{h} \phi_{h}\right\|_{H(r o t, \Omega)}^{2} \\
& \quad+\sum_{T \in \mathcal{T}_{h}} h_{T}^{2}\left\|\operatorname{div} \mathcal{C} \varepsilon\left(\phi_{h}\right)+\gamma_{h}+\frac{t^{2}}{12} \alpha_{t, h} \phi_{h}\right\|_{T}^{2}+\sum_{E \in \mathcal{E}_{h}} h_{E}\left\|\left[\mathcal{C} \varepsilon\left(\phi_{h}\right)\right]_{E} n_{E}\right\|_{E}^{2}+\mu_{h}\left(\gamma_{h}\right)^{2} \\
& \lesssim\left|\omega-\omega_{h}\right|_{1}^{2}+\left|\phi-\phi_{h}\right|_{1}^{2}+\left(1+\zeta^{-1} t^{2}\right) \zeta^{-1} t^{2}\left\|\gamma-\gamma_{h}\right\|^{2}+\left\|\gamma-\gamma_{h}\right\|_{-1} \\
& \quad+\zeta^{-2} t^{4}\left\|\operatorname{rot}\left(\gamma-\gamma_{h}\right)\right\|^{2}+\sum_{T \in \mathcal{T}_{h}} h_{T}^{2}\left(t^{2}+h_{T}^{2}\right)\left\|\alpha_{t} \omega-\alpha_{t, h} \omega_{h}\right\|_{T}^{2} \\
& \quad+\frac{t^{2}}{12} \sum_{T \in \mathcal{T}_{h}} h_{T}^{2}\left\|\alpha_{t} \phi-\alpha_{t, h} \phi_{h}\right\|_{T}^{2}+\left(\frac{t^{2}}{12}\left\|\alpha_{t} \phi-\alpha_{t, h} \phi_{h}\right\|\right)^{2} .
\end{aligned}
$$

Proof: The proof is a direct consequence of lemma 6.1, 6.2, 6.3 and 6.4.

Like the Corollaries 4.8 and 5.2, we have

Corollary 6.6 (Efficiency of the estimator) Assume that the eigenvalue $\alpha_{t}$ is simple, then

$$
\begin{aligned}
& \sum_{T \in \mathcal{T}_{h}} h_{T}^{2}\left(t^{2}+h_{T}^{2}\right)\left\|\alpha_{t, h} \omega_{h}+\operatorname{div} \gamma_{h}\right\|_{T}^{2}+\sum_{E \in \mathcal{E}_{h}} h_{E}\left(t^{2}+h_{E}^{2}\right)\left\|\left[\gamma_{h}\right]_{E} \cdot n_{E}\right\|_{E}^{2}+\left\|\phi_{h}-\boldsymbol{R}_{h} \phi_{h}\right\|_{H(r o t, \Omega)}^{2} \\
& \quad+\sum_{T \in \mathcal{T}_{h}} h_{T}^{2}\left\|\operatorname{div} \mathcal{C} \varepsilon\left(\phi_{h}\right)+\gamma_{h}+\frac{t^{2}}{12} \alpha_{t, h} \phi_{h}\right\|_{T}^{2}+\sum_{E \in \mathcal{E}_{h}} h_{E}\left\|\left[\mathcal{C} \varepsilon\left(\phi_{h}\right)\right]_{E} n_{E}\right\|_{E}^{2}+\mu_{h}\left(\gamma_{h}\right)^{2} \\
& \lesssim\left|\omega-\omega_{h}\right|_{1}^{2}+\left|\phi-\phi_{h}\right|_{1}^{2}+\left(1+\zeta^{-1} t^{2}\right) \zeta^{-1} t^{2}\left\|\gamma-\gamma_{h}\right\|^{2}+\left\|\gamma-\gamma_{h}\right\|_{-1} \\
& \quad+\zeta^{-2} t^{4}\left\|\operatorname{rot}\left(\gamma-\gamma_{h}\right)\right\|^{2}+\text { h.o.t. }
\end{aligned}
$$

\section{Numerical validation}

Here we illustrate and validate our theoretical results by a simple computational example. Let $\Omega$ be the unit square $] 0,1\left[{ }^{2}\right.$. We take $\nu=0.3, k=5 / 6$ and $t=0.1$. The meshes we use are uniform ones composed of $n^{2}$ squares, each of them being cut into 8 triangles as displayed the Figure 1 for $n=4$. The refinement strategy is an uniform one so that the value of the mesh size $h$ between two consecutive meshes is twice smaller.

Before evaluating the a posteriori error estimator, we compute $\omega_{t, h}^{1}$ by :

$$
\omega_{t, h}^{1}=\sqrt{2(1+\nu)} t \sqrt{\alpha_{t, h}^{1}}
$$

where $\alpha_{t, h}^{1}$ is the first computed approximated eigenvalue. In fact, this rescaling process is done in order to allow some comparisons with some bibliography data $[14,17,27]$. Table 1 displays the obtained values for different values of $n$, and shows that $\omega_{t, h}^{1}$ converges when $h$ goes towards zero, as theoretically expected. 


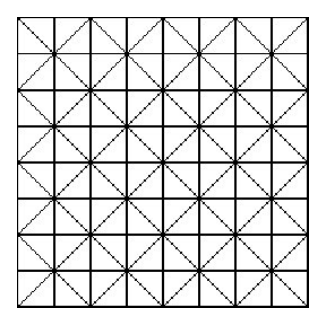

Figure 1: Mesh level corresponding to $n=4$ and $h=\sqrt{2} / 8$.

\begin{tabular}{|c||c|c|c|c|c|c|c|}
\hline$n$ & 2 & 4 & 8 & 16 & 32 & 64 & 128 \\
\hline$\omega_{t, h}^{1}$ & 2.3391 & 1.6992 & 1.6129 & 1.5934 & 1.5886 & 1.5874 & 1.5871 \\
\hline
\end{tabular}

Table 1: Values of the first approximated eigenvalue $\omega_{t, h}^{1}$.

Now, our result on the finest grid $(n=128)$ is compared in Table 2 with the ones obtained by the Huang and Hinton method in [27] (column HH), the Dawe and Roufaeil method in [14] (column DR) and the Durán, Hervella-Nieto, Liberman, Rodríguez and Solomin method in [17] (column DHLRS). Our value is clearly in good agreement with these references, even if from Table 2 it can noticed that it is the smallest one. This can be explained by the fact that our mesh resolution is finer. Indeed, in Table 3, it can be observed similar results for similar mesh resolutions.

\begin{tabular}{|c|c|c||c|}
\hline HH & DR & DHLRS & Our result \\
\hline 1.591 & 1.594 & 1.5913 & 1.587 \\
\hline
\end{tabular}

Table 2: The first value of $\omega_{t, h}^{1}$. (Value obtained with the finest mesh avalaible in each paper).

To verify the estimator reliability presented in section 5, the error estimator $\eta_{e v}$ is defined by :

$$
\eta_{e v}^{2}=\eta_{h, 1}^{2}+\eta_{h, 2}^{2}+\eta_{h, 3}^{2}+\eta_{h, 4}^{2}+\eta_{h, 5}^{2}+\eta_{h, 6}^{2},
$$

where the different contributions are given by :

$$
\begin{aligned}
\eta_{h, 1}^{2} & =\sum_{T \in \mathcal{T}_{h}} h_{T}^{2}\left(t^{2}+h_{T}^{2}\right)\left\|\alpha_{t, h} \omega_{h}+\operatorname{div} \gamma_{h}\right\|_{T}^{2}, \\
\eta_{h, 2}^{2} & =\sum_{E \in \mathcal{E}_{h}} h_{E}\left(t^{2}+h_{E}^{2}\right)\left\|\left[\gamma_{h}\right]_{E} \cdot n_{E}\right\|_{E}^{2}, \\
\eta_{h, 3}^{2} & =\sum_{T \in \mathcal{T}_{h}} h_{T}^{2}\left\|\operatorname{div} \mathcal{C} \varepsilon\left(\phi_{h}\right)+\gamma_{h}+\frac{t^{2}}{12} \alpha_{t, h} \phi_{h}\right\|_{T}^{2},
\end{aligned}
$$




\begin{tabular}{|c||c||c|c|c|c|c|}
\cline { 2 - 8 } \multicolumn{1}{c|}{} & \multicolumn{1}{c||}{$n$} & 5 & 10 & \multicolumn{2}{c|}{20} & \multicolumn{1}{c|}{} \\
\cline { 2 - 8 } \multicolumn{1}{c|}{} & DHLRS & 1.5947 & 1.5921 & 1.5913 & \multicolumn{1}{c|}{} \\
\hline$n$ & 4 & 8 & 16 & 32 & 64 & 128 \\
\hline Our result & 1.699 & 1.613 & 1.593 & 1.589 & 1.587 & 1.587 \\
\hline
\end{tabular}

Table 3: The first value of $\omega_{t, h}^{1}$. Comparison with DHLRS for different mesh resolutions.

$$
\begin{aligned}
\eta_{h, 4}^{2} & =\sum_{E \in \mathcal{E}_{h}} h_{E}\left\|\left[\mathcal{C} \varepsilon\left(\phi_{h}\right)\right]_{E} n_{E}\right\|_{E}^{2}, \\
\eta_{h, 5}^{2} & =\left\|\phi_{h}-\mathbf{R}_{h} \phi_{h}\right\|_{H(r o t, \Omega)}^{2} \\
\eta_{h, 6}^{2} & =\mu_{h}^{2}\left(\gamma_{h}\right) .
\end{aligned}
$$

We plot in Figure 2 the evolution of the computed estimator $\eta_{e v}$ as well as its different contributions $\eta_{h, i}, i=1 . .6$ versus $h$. First, it can be seen that the contributions $\eta_{h, 4}$ and $\eta_{h, 6}$ converge at order 3 and that the contributions $\eta_{h, 3}$ and $\eta_{h, 5}$ converge at order 2 . Moreover, it is clear that the main part of $\eta_{e v}$ is $\eta_{h, 2}$. Nevertheless, we can also remark that the convergence rate of $\eta_{h, 2}$ (resp. $\eta_{h, 1}$ ) starts for coarse meshes near to 2 (resp. 3). This behaviour can easily be explained by the definition of $\eta_{h, 2}$ (resp. $\eta_{h, 1}$ ) when $h$ is larger than $t$. As soon as $h$ becomes smaller than $t$, the convergence rate equal to 1 for $\eta_{h, 2}$ (resp. 2 for $\left.\eta_{h, 1}\right)$ is recovered as it can be observed in Figure 2 for the finest meshes.

\section{Acknowledgements}

The authors are grateful to the referee for her/his helpful comments and suggestions which allowed to improve the obtained results. 


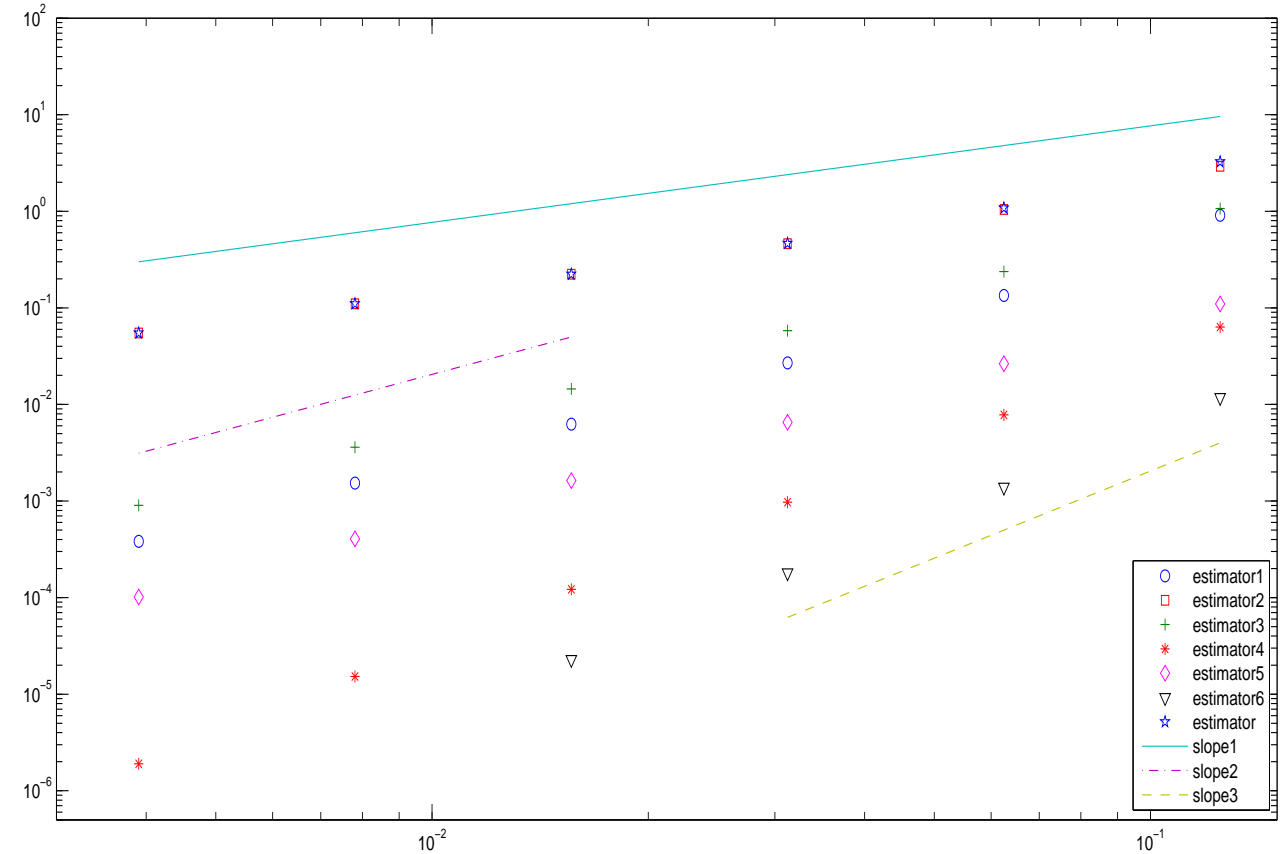

Figure 2: Estimators convergence rate, $t=0.1$. 


\section{References}

[1] M. Ainsworth and J. T. Oden. A posteriori error estimation in finite element analysis. Pure and Applied Mathematics (New York). Wiley-Interscience [John Wiley \& Sons], New York, 2000.

[2] D. N. Arnold and R.S. Falk. A uniformly accurate finite element method for the Reissner-Mindlin plate. SIAM J. Numer. Anal., 26(6):1276-1290, 1989.

[3] I. Babuška and J. Osborn. Eigenvalue problems. In Handbook of numerical analysis, Vol. II, Handb. Numer. Anal., II, pages 641-787. North-Holland, Amsterdam, 1991.

[4] I. Babuška and T. Strouboulis. The finite element method and its reliability. Numerical Mathematics and Scientific Computation. The Clarendon Press Oxford University Press, New York, 2001.

[5] K.-J. Bathe and E. N. Dvorkin. A four-node plate bending element based on Mindlin-Reissner plate theory and a mixed interpolation. Internat. J. Numer. Methods Engrg., 21:367-383, 1985.

[6] L. Beirão da Veiga, C. Chinosi, C. Lovadina, and R. Stenberg. A-priori and a-posteriori error analysis for a family of Reissner-Mindlin plate elements. BIT, 48(2):189-213, 2008.

[7] F. Brezzi, K.-J. Bathe, and M. Fortin. Mixed-interpolated elements for Reissner-Mindlin plates. Internat. J. Numer. Methods Engrg., 28(8):1787-1801, 1989.

[8] F. Brezzi and M. Fortin. Mixed and hybrid finite element methods, volume 15 of Springer Series in Computational Mathematics. Springer-Verlag, New York, 1991.

[9] C. Carstensen. Residual-based a posteriori error estimate for a nonconforming Reissner-Mindlin plate finite element. SIAM J. Numer. Anal., 39(6):2034-2044 (electronic), 2002.

[10] C. Carstensen and J. Hu. A posteriori error analysis for conforming MITC elements for ReissnerMindlin plates. Math. Comp., 77(262):611-632, 2008.

[11] C. Carstensen and J. Schöberl. Residual-based a posteriori error estimate for a mixed Reißner-Mindlin plate finite element method. Numer. Math., 103(2):225-250, 2006.

[12] E. Creusé, S. Nicaise, and E. Verhille. Robust equilibrated a posteriori error estimators for the Reissner-Mindlin system. Calcolo, 48(4):307-335, 2011.

[13] M. Dauge. Elliptic boundary value problems on corner domains - smoothness and asymptotics of solutions, volume 1341 of Lecture Notes in Mathematics. Springer, Berlin, 1988.

[14] D. J. Dawe and O. L. Roufaeil. Rayleigh-Ritz vibration analysis of Mindlin plates. Journal of Sound and Vibration, 69(3):345-359, 1980.

[15] R. Durán and E. Liberman. On mixed finite element methods for the Reissner-Mindlin plate model. Math. Comp., 58(198):561-573, 1992.

[16] R. G. Durán, E. Hernández, L. Hervella-Nieto, E. Liberman, and R. Rodríguez. Error estimates for low-order isoparametric quadrilateral finite elements for plates. SIAM J. Numer. Anal., 41(5):17511772 (electronic), 2003.

[17] R. G. Durán, L. Hervella-Nieto, E. Liberman, R. Rodríguez, and J. Solomin. Approximation of the vibration modes of a plate by Reissner-Mindlin equations. Math. Comp., 68(228):1447-1463, 1999.

[18] R. G. Durán, L. Hervella-Nieto, E. Liberman, R. Rodríguez, and J. Solomin. Finite element analysis of the vibration problem of a plate coupled with a fluid. Numer. Math., 86(4):591-616, 2000.

[19] R.G. Durán, C. Padra, and R. Rodríguez. A posteriori error estimates for the finite element approximation of eigenvalue problems. Math. Models Methods Appl. Sci., 13(8):1219-1229, 2003. 
[20] M. E. Frolov, P. Neittaanmäki, and S. I. Repin. Guaranteed functional error estimates for the ReissnerMindlin plate problem. J. Math. Sci. (N. Y.), 132(4):553-561, 2006. Problems in mathematical analysis. No. 31.

[21] V. Girault and P.-A. Raviart. Finite element methods for Navier-Stokes equations, volume 5 of Springer Series in Computational Mathematics. Springer-Verlag, Berlin, 1986. Theory and algorithms.

[22] D. Gómez, M. Lobo, and E. Pérez. On the vibrations of a plate with a concentrated mass and very small thickness. Math. Methods Appl. Sci., 26(1):27-65, 2003.

[23] P. Grisvard. Singularité en elasticité. Archive for Rational Mechanics and Analysis, 107:157-180, 1989.

[24] P. Grisvard. Singularities in boundary value problems, volume 22 of Recherches en Mathématiques Appliquées [Research in Applied Mathematics]. Masson, Paris, 1992.

[25] E. Hernández. Approximation of the vibration modes of a plate coupled with a fluid by low-order isoparametric finite elements. M2AN Math. Model. Numer. Anal., 38(6):1055-1070, 2004.

[26] J. Hu and Y. Huang. A posteriori error analysis of finite element methods for Reissner-Mindlin plates. SIAM J. Numer. Anal., 47(6):4446-4472, 2010.

[27] H.C. Huang and E. Hinton. A nine node Lagrangian Mindlin plate element with enhanced shear interpolation. Eng. Comput., 1(4):369-379, 1984.

[28] T. Kato. Perturbation theory for linear operators. Classics in Mathematics. Springer-Verlag, Berlin, 1995. Reprint of the 1980 edition.

[29] M.G. Larson. A posteriori and a priori error analysis for finite element approximations of self-adjoint elliptic eigenvalue problems. SIAM J. Numer. Anal., 38(2):608-625 (electronic), 2000.

[30] E. Liberman. A posteriori error estimator for a mixed finite element method for Reissner-Mindlin plate. Math. Comp., 70(236):1383-1396 (electronic), 2001.

[31] C. Lovadina, D. Mora, and R. Rodríguez. Approximation of the buckling problem for Reissner-Mindlin plates. SIAM J. Numer. Anal., 48(2):603-632, 2010.

[32] C. Lovadina and R. Stenberg. A posteriori error analysis of the linked interpolation technique for plate bending problems. SIAM J. Numer. Anal., 43(5):2227-2249 (electronic), 2005.

[33] P. Neittaanmäki and S. Repin. Reliable methods for computer simulation, volume 33 of Studies in Mathematics and its Applications. Elsevier Science B.V., Amsterdam, 2004. Error control and a posteriori estimates.

[34] S. Nicaise. Regularity of the solutions of elliptic systems in polyhedral domains. Bulletin Belgium Math. Soc.-S. Stevin, 4:411-429, 1997.

[35] R. Stenberg and M. Suri. An $h p$ error analysis of MITC plate elements. SIAM J. Numer. Anal., $34(2): 544-568,1997$.

[36] R. Verfürth. A review of a posteriori error estimation and adaptive mesh-refinement techniques. Wiley-Teubner Series Advances in Numerical Mathematics. Wiley-Teubner, Chichester, Stuttgart, 1996. 